\title{
ON INTERPOLATION APPROXIMATION: CONVERGENCE RATES FOR POLYNOMIAL INTERPOLATION FOR FUNCTIONS OF LIMITED REGULARITY*
}

\author{
SHUHUANG XIANG ${ }^{\dagger}$
}

\begin{abstract}
The convergence rates on polynomial interpolation in most cases are estimated by Lebesgue constants. These estimates may be overestimated for some special points of sets for functions of limited regularities. In this paper, by applying the Peano kernel theorem and Wainerman's lemma, new formulas on the convergence rates are considered. Based upon these new estimates, it shows that the interpolation at strongly normal pointsystems can achieve the optimal convergence rate, the same as the best polynomial approximation. Furthermore, by using the asymptotics on Jacobi polynomials, the convergence rates are established for Gauss-Jacobi, Jacobi-Gauss-Lobatto or Jacobi-Gauss-Radau pointsystems. From these results, we see that the interpolations at the Gauss-Legendre, Legendre-Gauss-Lobatto pointsystem, or at strongly normal pointsystems, has essentially the same approximation accuracy compared with those at the two Chebyshev piontsystems, which also illustrates the equally accuracy of the Gauss and Clenshaw-Curtis quadrature. In addition, numerical examples illustrate the perfect coincidence with the estimates, which means the convergence rates are optimal.
\end{abstract}

Key words. polynomial interpolation, Peano kernel, convergence rate, limited regularity, strongly normal pointsystem, Gauss-Jacobi point, Jacobi-Gauss-Lobatto point, Chebyshev point.

AMS subject classifications. 65D05, 65D25

1. Introduction. A central problem in approximation theory is the construction of simple functions that are easily implemented on computers and approximate well a given set of functions.

There exist many investigations for the behavior of continuous functions approximated by polynomials. Weierstrass 73 in 1885 proved the well known result that every continuous function $f(x)$ in $[-1,1]$ can be uniformly approximated as closely as desired by a polynomial function. This result has both practical and theoretical relevance, especially in polynomial interpolation.

Polynomial interpolation is a fundamental tool in many areas of scientific computing. Lagrange interpolation is a classical technique for approximation of continuous functions. Let us denote by

$$
-1 \leq x_{n}^{(n)}<x_{n-1}^{(n)}<\cdots<x_{2}^{(n)}<x_{1}^{(n)} \leq 1
$$

the $n$ distinct points in the interval $[-1,1]$ and let $f(x)$ be a function defined in the same interval. The $n$th Lagrange interpolation polynomial of $f(x)$ is unique and given by the formula

$$
L_{n}[f]=\sum_{k=1}^{n} f\left(x_{k}^{(n)}\right) \ell_{k}^{(n)}(x), \quad \ell_{k}^{(n)}(x)=\frac{\omega_{n}(x)}{\omega_{n}^{\prime}\left(x_{k}^{(n)}\right)\left(x-x_{k}^{(n)}\right)},
$$

where $\omega_{n}(x)=\left(x-x_{1}^{(n)}\right)\left(x-x_{2}^{(n)}\right) \cdots\left(x-x_{n}^{(n)}\right)$.

There is a well developed theory that quantifies the convergence or divergence of the Lagrange interpolation polynomials (Brutman [7, 8] and Trefethen [59]). Two key notions for interpolation in a given set of points are that of the Lebesgue function

$$
\lambda_{n}(x)=\sum_{k=1}^{n}\left|\ell_{k}^{(n)}(x)\right|
$$

and Lebesgue constant

$$
\Lambda_{n}=\max _{x \in[-1,1]} \lambda_{n}(x),
$$

\footnotetext{
${ }^{*}$ This work was supported by National Science Foundation of China (No. 11371376).

${ }^{\dagger}$ School of Mathematics and Statistics, Central South University, Changsha, Hunan 410083, P. R. China.
} 
which are of fundamental importance (Cheney [9], Davis [12] and Szegö [55]). The Lebesgue constant can also be interpreted as the $\infty$-norm of the projection operator $L_{n}: C([-1,1]) \rightarrow$ $\mathcal{P}_{n-1}$

$$
\Lambda_{n}=\sup _{f} \frac{\left\|L_{n}[f]\right\|_{\infty}}{\|f\|_{\infty}}
$$

where $\mathcal{P}_{n-1}$ is the set of polynomials of degree less than or equal to $n-1$.

Based upon the Lebesgue constant, the interpolation error can be estimated by

$$
\left\|L_{n}[f]-f\right\|_{\infty} \leq\left(1+\Lambda_{n}\right)\left\|p_{n-1}^{*}-f\right\|_{\infty},
$$

where $p_{n-1}^{*}$ is the best polynomial approximation of degree $n-1$. Thus, the Lebesgue constant $\Lambda_{n}$ indicates how good the interpolant $L_{n}[f]$ is in comparison with the best polynomial approximation $p_{n-1}^{*}$.

The study of the Lebesgue constant $\Lambda_{n}$ originated more than 100 years ago. Comprehensive reviews can be found in Brutman [8, Lubinsky [41, Trefethen [59, Chapter 15], etc. For an arbitrarily given system of points $\left\{x_{1}^{(n)}, x_{2}^{(n)}, \ldots, x_{n}^{(n)}\right\}_{n=1}^{\infty}$, Bernstein [2] and Faber [18] in 1914 obtained that

$$
\Lambda_{n} \geq \frac{1}{12} \log n,
$$

which, together with the boundedness principle, implies that there exists a continuous function $f(x)$ in $[-1,1]$ for which the sequence $L_{n}[f](n=1,2, \ldots)$ is not uniformly convergent to $f$ in $[-1,1]$. More precisely, Erdös [15] and Brutman [7] proved that

(1.6) $\Lambda_{n} \geq \frac{2}{\pi} \log n+C$ for some constant $C([15]) ; \quad \Lambda_{n} \geq \frac{2}{\pi}\left(\gamma_{0}+\log \frac{4}{\pi}\right)+\frac{2}{\pi} \log n([7])$,

where $\gamma_{0}=0.577 \ldots$ is the Euler's constant. In particular, for equidistant pointsystem

$$
\left\{x_{k}^{(n)}=-1+\frac{2 k}{n-1}\right\}_{k=0}^{n-1},
$$

Schönhage [52] showed that

$$
\Lambda_{n} \sim \frac{2^{n}}{e\left(\log (n-1)+\gamma_{0}\right)(n-1)}, \quad n \rightarrow \infty .
$$

Additionally, Trefethen and Weideman 61 established that

$$
\frac{2^{n-3}}{(n-1)^{2}} \leq \Lambda_{n} \leq \frac{2^{n+2}}{n-1}, \quad n \geq 0 .
$$

Then generally, the set of equally spaced points is a bad choice for Lagrange interpolation (see Runge [49).

Whereas, for well chosen sets of points, the growth of $\Lambda_{n}$ may be extremely slow as $n \rightarrow \infty$ :

- Chebyshev pointsystem of first kind $T_{n}=\left\{x_{k}^{(n)}=\cos \left(\frac{2 k-1}{2 n} \pi\right)\right\}_{k=1}^{n}$ : An asymptotic estimate of $\Lambda_{n}\left(T_{n}\right)$ was given by Bernstein [1] as

$$
\Lambda_{n}\left(T_{n}\right) \sim \frac{2}{\pi} \log n, \quad n \rightarrow \infty,
$$

\footnotetext{
${ }^{1}$ Grünwald 24] in 1935 and Marcinkiewicz [4] in 1937, independently, showed that even for the Chebyshev points of first kind

$$
x_{k}^{(n)}=\cos \left(\frac{2 k-1}{2 n} \pi\right), \quad k=1,2, \ldots, n, \quad n=1,2, \ldots,
$$

there is a continuous function $f(x)$ in $[-1,1]$ for which the sequence $L_{n}[f]$ is divergent everywhere in $[-1,1]$.
} 
which is improved by Ehlich and Zeller [14], Rivlin [47] and Brutman [7] as

$$
\frac{2}{\pi}\left(\gamma_{0}+\log \frac{4}{\pi}\right)+\frac{2}{\pi} \log n<\Lambda_{n}\left(T_{n}\right) \leq 1+\frac{2}{\pi} \log n, \quad n=1,2, \ldots
$$

- Chebyshev pointsystem of second kind $U_{n}=\left\{x_{k}^{(n)}=\cos \left(\frac{k}{n-1} \pi\right)\right\}_{k=0}^{n-1}$ (also called Chebyshev extreme or Clenshaw-Curtis points [58]): Ehlich and Zeller [14] proved that

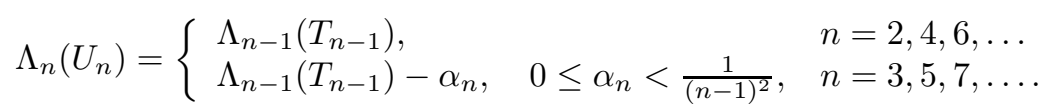

- The roots of Jacobi polynomial $P_{n}^{(\alpha, \beta)}(x)(\alpha, \beta>-1)$ : The asymptotic estimate of $\Lambda_{n}\left(J_{n}\right)$ was found by Szegö [55] as

$$
\Lambda_{n}\left(J_{n}\right)=\left\{\begin{array}{ll}
O\left(n^{\gamma+\frac{1}{2}}\right), & \gamma>-\frac{1}{2} \\
O(\log n), & \gamma \leq-\frac{1}{2}
\end{array}, \quad \gamma=\max \{\alpha, \beta\} .\right.
$$

Comparing Equations (1.7), (1.8) and (1.9) with (1.6), we see that the two Chebyshev pointsystems and the Jacobi pointsystem with $\gamma \leq-\frac{1}{2}$ are nearly optimal and of order $O(\log n)$.

Nevertheless, it is worth noting that if $f(x)$ has an absolutely continuous $(k-1)$ st derivative $f^{(k-1)}$ on $[-1,1]$ for some $k \geq 1$ and its $k$-th derivative $f^{(k)}$ is of bounded variation $\operatorname{Var}\left(f^{(k)}\right)<$ $\infty$, Mastroianni and Szabados [42], Trefethen [59] and Xiang et al. [76] proved that

$$
\left\|f-L_{n}[f]\right\|_{\infty}=O\left(n^{-k}\right),
$$

where $L_{n}[f]$ is at the $n$ Chebyshev points of first or second kind, which has the same asymptotic order as $\left\|f-p_{n-1}^{*}\right\|_{\infty}$ for the best approximation $p_{n-1}^{*}$, following de la Vallée Poussin [62]. In particular, for $f(x)=|x|$, the error on the $L_{n}[f]$ at the above two Chebyshev pointsystems satisfies

$$
\left\|f-L_{n}[f]\right\|_{\infty} \leq \frac{4}{\pi(n-1)}
$$

(see [59, 76]), while

$$
\left\|f-p_{n-1}^{*}\right\|_{\infty} \sim \frac{\beta}{n}, \quad 0.2801685<\beta<0.2801734
$$

(see Bernstein [3] and Varga and Capenter 63]). Thus, the error estimate (1.5) by using the Lebesgue constant may be overestimated for some special points of sets for functions of limited regularities.

Moreover, it has been observed, by Clenshaw-Curtis [10] and O'Hara and Smith [29, that $n$-point Gauss quadrature and $n$-point Clenshaw-Curtis quadrature have essentially the same accuracy, which has been showed recently by Trefethen [58, 59, Brass and Petras [6] and Xiang and Bornemann [75]. Both of these two quadrature are derived from the interpolation polynomial $L_{n}[f]$ by

$$
Q_{n}[f]=\int_{-1}^{1} L_{n}[f](x) d x
$$

based on the $n$ Gauss-Legender and Clenshaw-Curtis points, respectively. From this observation, we may conclude that the corresponding interpolation $L_{n}[f]$ based on these two pointsystems may have the same convergence rate. However, it can not be derived from (1.5).

In this paper, we present new convergence rates of the interpolation polynomials for functions of limited regularities, based upon the famous Peano kernel theorem [45] and applying an interesting Wainerman's lemma [72. Suppose $f(x)$ has an absolutely continuous $(r-1)$ st 
derivative $f^{(r-1)}$ on $[-1,1]$, and its $r$-th derivative $f^{(r)}$ is of bounded variation $\operatorname{Var}\left(f^{(r)}\right)<\infty$. We will show that

$$
\left\|f-L_{n}[f]\right\|_{\infty} \leq \frac{\pi^{r} \operatorname{Var}\left(f^{(r)}\right)}{(n-1)(n-2) \cdots(n-r)} \max _{1 \leq j \leq n}\left\|\ell_{j}^{(n)}\right\|_{\infty},
$$

which leads to

$$
\left\|f-L_{n}[f]\right\|_{\infty}=O\left(n^{-r} \max _{1 \leq j \leq n}\left\|\ell_{j}^{(n)}\right\|_{\infty}\right)
$$

The Lebesgue constant $\Lambda_{n}=\max _{x \in[-1,1]} \sum_{k=1}^{n}\left|\ell_{k}^{(n)}(x)\right|$ is replaced by $\max _{1 \leq j \leq n}\left\|\ell_{j}^{(n)}\right\|_{\infty}$ in some sense since $\left\|f-p_{n-1}^{*}\right\|_{\infty}=O\left(n^{-r}\right)[62$.

Particularly, from (1.12), it directly follows that the interpolation $L_{n}[f]$ at a strongly normal pointsystem (see Fejér [19]) can achieve the optimal convergence rate as $O\left(\left\|f-p_{n-1}^{*}\right\|_{\infty}\right)$.

Furthermore, $\left\|\ell_{j}\right\|_{\infty}$ can be explicitly estimated for Gauss-Jacobi, Jacobi-Gauss-Lobatto or Jacobi-Gauss-Radau pointsystems, by using the asymptotics on Jacobi polynomials given by Szegö [55] and some results given in Kelzon [34, 35], Vértesi [66, 68, Sun [54, Prestin [46], Kvernadze [38, Vecchia et al. 69, etc., as follows

- For the $n$ Gauss-Jacobi points:

$$
\max _{1 \leq j \leq n}\left\|\ell_{j}^{(n)}\right\|_{\infty}=O\left(n^{\max \left\{\gamma-\frac{1}{2}, 0\right\}}\right), \quad \gamma=\max \{\alpha, \beta\} .
$$

- For the $n$ Jacobi-Gauss-Lobatto points (the roots of $\left.\left(1-x^{2}\right) P_{n-2}^{(\alpha, \beta)}(x)=0\right)$ :

$$
\max _{1 \leq j \leq n}\left\|\ell_{j}^{(n)}\right\|_{\infty}=\left\{\begin{array}{ll}
O\left(n^{-\min \left\{0, \alpha+\frac{1}{2}, \beta+\frac{1}{2}\right\}}\right), & -1<\alpha, \beta \leq \frac{3}{2} \\
O\left(n^{-\min \left\{0, \alpha+\frac{1}{2}, 2+\alpha-\beta, \frac{5}{2}-\beta\right\}}\right), & -1<\alpha \leq \frac{3}{2}, \beta>\frac{3}{2} \\
O\left(n^{-\min \left\{0, \beta+\frac{1}{2}, 2+\beta-\alpha, \frac{5}{2}-\alpha\right\}}\right), & \alpha>\frac{3}{2},-1<\beta \leq \frac{3}{2} \\
O\left(n^{-\min \left\{0,2+\alpha-\beta, 2+\beta-\alpha, \frac{5}{2}-\alpha, \frac{5}{2}-\beta\right\}}\right), & \alpha, \beta>\frac{3}{2}
\end{array} .\right.
$$

- For the $n$ Jacobi-Gauss-Radau points $(1-x) P_{n-1}^{(\alpha, \beta)}(x)$

$$
\max _{0 \leq j \leq n-1}\left\|\ell_{j}^{(n)}\right\|_{\infty}=\left\{\begin{array}{ll}
O\left(n^{-\min \left\{0, \alpha+\frac{1}{2}, \alpha-\beta\right\}}\right), & -1<\alpha \leq \frac{1}{2} \\
O\left(n^{-\min \left\{0, \frac{1}{2}-\beta, \frac{5}{2}-\alpha, \alpha-\beta\right\}}\right), & \alpha>\frac{1}{2}
\end{array} .\right.
$$

- For the $n$ Jacobi-Gauss-Radau points $(1+x) P_{n-1}^{(\alpha, \beta)}(x)$

$$
\max _{1 \leq j \leq n}\left\|\ell_{j}^{(n)}\right\|_{\infty}=\left\{\begin{array}{ll}
O\left(n^{-\min \left\{0, \beta+\frac{1}{2}, \beta-\alpha\right\}}\right), & -1<\beta \leq \frac{1}{2} \\
O\left(n^{-\min \left\{0, \frac{1}{2}-\alpha, \frac{5}{2}-\beta, \beta-\alpha\right\}}\right), & \beta>\frac{1}{2}
\end{array} .\right.
$$

From the above estimates, we see that the interpolation at the Gauss-Legendre or at the Legendre-Gauss-Lobatto pointsystem, has essentially the same approximation accuracy compared with those at the two Chebyshev piontsystems. All of them satisfy that $\max _{1 \leq j \leq n}\left\|\ell_{j}^{(n)}\right\|_{\infty}=$ $O(1)$ (for more general cases see FIG. 1.1). In addition, the convergence rate is attainable illustrated by some functions of limited regularities.

Thus, the best approximation polynomial is challenged by the interpolation polynomials at the special pointsystems showed in FIG. 1.1. Furthermore, we will see that the interpolation polynomials at the special pointsystems perform much better than the best approximation polynomial for approximation the derivatives $f^{\prime}$ and $f^{\prime \prime}$ by $L_{n}^{\prime}[f], L_{n}^{\prime \prime}[f],\left[p_{n-1}^{*}\right]^{\prime}$ and $\left[p_{n-1}^{*}\right]^{\prime \prime}$, respectively, illustrated by numerical examples in the final section. 

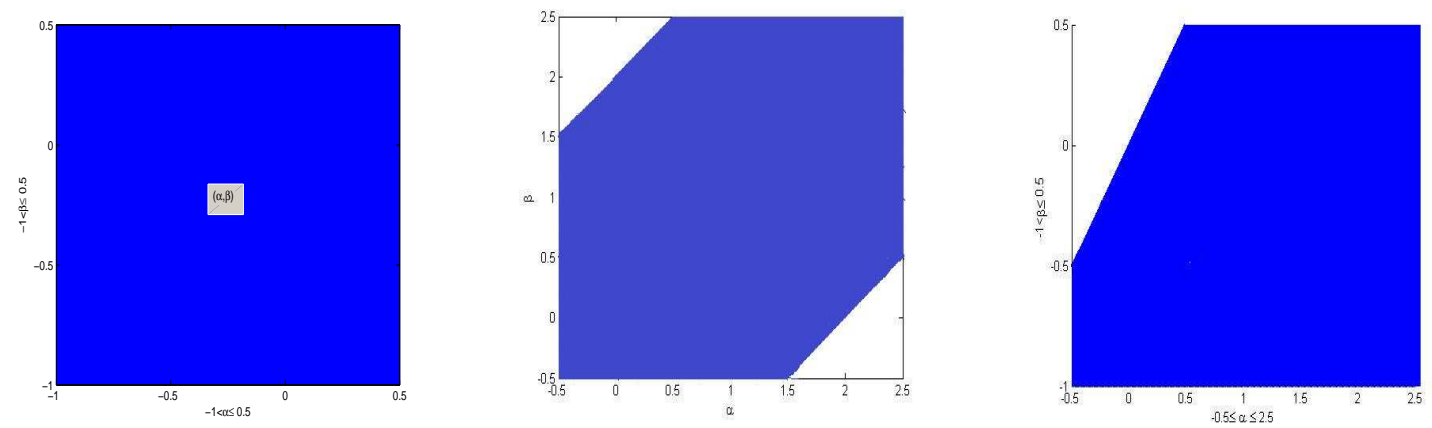

FIG. 1.1. The neighbourhood on $(\alpha, \beta)$ such that $\left\|f-L_{n}[f]\right\|_{\infty}=O\left(n^{-r}\right)$ for the Gauss-Jacobi pointsystems (left), Jacobi-Gauss-Lobatto pointsystems (middle) and Jacobi-Gauss-Radau $\left((1-x) P_{n-1}^{(\alpha, \beta)}(x)=0\right)$ pointsystems (right), respectively: $\operatorname{Var}\left(f^{(r)}\right)<\infty$.

It is worthy of special mention that the interpolation polynomial $L_{n}[f]$, at the GaussJacobi, Jacobi-Gauss-Lobatto or Gauss-Jacobi-Radau pointsystem, can be efficiently evaluated by applying the second barycentric formula

$$
L_{n}[f](x)=\frac{\sum_{j=1}^{n} \frac{\lambda_{j}}{x-x_{j}} f\left(x_{j}\right)}{\sum_{j=1}^{n} \frac{\lambda_{j}}{x-x_{j}}},
$$

which is robust in the presence of rounding errors 33 and costs overall computational complexity $O(n)$ [4, where the nodes $x_{j}$ and the barycentric weights $\lambda_{j}$ are computed by jacpts and the formulas given in 28, 70, 71], respectively. A MATLAB routine jacpts, which uses the algorithm in [26] for the computation of these nodes and weights, can be found in CHEBFun system [60]. For more details on this topic, see Salzer [50, Henrich [30, Berrut and Trefethen [4, Higham 32, 33, Glaser et al. 23, Wang and Xiang [70, Bogaert et al. 5, Hale and Trefethen 28, Hale and Townsend 26, Trefethen [59, Wang et. al. [71, etc. MATLAB routines can be found in Chebfun system [60] and Xiang and He [77.

The paper is organized as follows: In section 2, we present the error of $f(x)-L_{n}[f](x)$ for each fixed $x \in[-1,1]$ by using the Peano representation and the bounded variation. In section 3, we introduce the interesting Wainerman's lemma and deduce the error bound on $\left\|f-L_{n}[f]\right\|_{\infty}$ by $\max _{1 \leq j \leq n}\left\|\ell_{j}^{(n)}\right\|_{\infty}$. We consider, in section 4 , the estimates of $\left\|\ell_{j}^{(n)}\right\|_{\infty}$ and derive the convergence rates for the interpolation polynomial at strongly normal pointsystems, Gauss-Jacobi, Jacobi-Gauss-Lobatto and Jacobi-Gauss-Radau pointsystems, respectively, where the convergence rates and attainability are illustrated by numerical experiments.

Throughout this paper, $A \sim B$ means that there exist positive constants $C_{1}$ and $C_{2}$ such that

$$
C_{1} B \leq A \leq C_{2} B
$$

For simplicity, in the following we abbreviate $x_{k}^{(n)}$ as $x_{k}$ and $\ell_{k}^{(n)}(x)$ as $\ell_{k}(x)$.

All the numerical results in this paper are carried out by using MATLAB R2012a on a desktop (2.8 GB RAM, 2 Core2 (32 bit) processors at $2.80 \mathrm{GHz}$ ) with Windows XP operating system.

2. The Peano kernel theorem. There are two general methods for deriving strict error bounds (Dahlquist and Björck [11). One applies the norms and distance formula together with the Lebesgue constants, which often overestimates the error. The other is due to the Peano kernel theorem.

Suppose $\mathcal{L}$ a continuously linear functional that maps functions $f \in C([-1,1])$ to $R$ satisfying $\mathcal{L}\left(f_{1}+f_{2}\right)=\mathcal{L} f_{1}+\mathcal{L} f_{2}$ for any $f_{1}, f_{2} \in C([-1,1])$ and $\mathcal{L}(\alpha f)=\alpha \mathcal{L} f$ for any scalar $\alpha$. 
In addition, we assume $\mathcal{L}\left[\mathcal{P}_{r-1}\right]=\{0\}$ for some $r \in\{1,2, \ldots\}$, where $\mathcal{P}_{r-1}$ denotes the set of polynomials with degree less than or equal to $r-1$.

The Peano kernel theorem (Peano [45, see also Kowalewski [36], Schmidt [51] and Mises [44]) is the identity

$$
\mathcal{L}[f]=\int_{-1}^{1} f^{(r)}(t) K_{r}(t) d t
$$

holding for all such functions $f \in C^{r}([-1,1])$, where $K_{r}(t)=\frac{1}{(r-1) !} \mathcal{L}\left[(x-t)_{+}^{r-1}\right]$ and

$$
(x-t)_{+}^{r-1}=\left\{\begin{array}{ll}
(x-t)^{r-1}, & x \geq t \\
0, & x<t
\end{array} \quad(r \geq 2), \quad(x-t)_{+}^{0}=\left\{\begin{array}{ll}
1, & x \geq t \\
0, & x<t
\end{array} \quad(r=1)\right.\right.
$$

For each fixed $x \in[-1,1]$, we consider the special functional $\mathcal{L}=E_{n}$, where $E_{n}[f](x)$ is defined for $\forall f \in C([-1,1])$ by

$$
E_{n}[f](x)=f(x)-\sum_{j=1}^{n} f\left(x_{j}\right) \ell_{j}(x)=f(x)-L_{n}[f](x)
$$

with $-1 \leq x_{n}<x_{n-1}<\cdots<x_{2}<x_{1} \leq 1$. $E_{n}[f]$ is a continuously linear functional since $\left|E_{n}[f](x)-E_{n}[g](x)\right| \leq\left(1+\Lambda_{n}\right)\|f-g\|_{\infty}$ for arbitrary $f, g \in C([-1,1])$, and then by the Peano theorem [45] $E_{n}[f]$ can be represented if $f \in C^{r}([-1,1])$ for $n \geq r$ as

$$
E_{n}[f](x)=\int_{-1}^{1} f^{(r)}(t) K_{r}(t) d t
$$

with

$$
K_{r}(t)=\frac{1}{(r-1) !}(x-t)_{+}^{r-1}-\frac{1}{(r-1) !} \sum_{j=1}^{n}\left(x_{j}-t\right)_{+}^{r-1} \ell_{j}(x) .
$$

Particularly, from (2.3) it implies

$$
\left|E_{n}[f](x)\right| \leq\left\|f^{(r)}\right\|_{\infty} \int_{-1}^{1}\left|K_{r}(t)\right| d t \leq 2\left\|f^{(r)}\right\|_{\infty}\left\|K_{r}\right\|_{\infty}
$$

Similar to the Peano kernel for quadrature 6], the kernel for interpolation satisfies the following proposition.

Proposition 2.1. (Peano representation) Let

$$
K_{s}(t)=\frac{1}{(s-1) !}(x-t)_{+}^{s-1}-\frac{1}{(s-1) !} \sum_{j=1}^{n}\left(x_{j}-t\right)_{+}^{s-1} \ell_{j}(x), \quad s=1,2, \ldots
$$

Then for $s \geq 2$, the Peano kernel satisfies $K_{s}(-1)=K_{s}(1)=0$ and can be rewritten as

$$
K_{s}(u)=\int_{u}^{1} K_{s-1}(t) d t, \quad s=2,3, \ldots
$$

Proof. From the definition of $K_{s}$ in $(2.5)$, it is easy to verify that $K_{s}(-1)=K_{s}(1)=0$ by using $\sum_{j=1}^{n} \ell_{j}(t) \equiv 1$ for $t \in[-1,1]$. Furthermore, we find that

$$
\begin{aligned}
(2.7)^{\frac{1}{(s-2) !} \int_{u}^{1}(x-t)_{+}^{s-1} d t} & = \begin{cases}0, & u>x \\
\frac{1}{(s-2) !} \int_{u}^{x}(x-t)^{s-1} d t=\frac{1}{(s-1) !}(x-u)^{s-1}, & u \leq x\end{cases} \\
& =\frac{1}{(s-1) !}(x-u)_{+}^{s-1} .
\end{aligned}
$$


Define $x_{0}=1$ and $x_{n+1}=-1$ and suppose $x_{m+1}<u \leq x_{m}$ for some nonnegative integer $m$. By (2.7), similarly we have

$$
\begin{aligned}
\frac{1}{(s-2) !} \sum_{j=1}^{n} \int_{u}^{1}\left(x_{j}-t\right)_{+}^{s-2} \ell_{j}(x) d t & =\frac{1}{(s-2) !} \sum_{j=1}^{m} \ell_{j}(x) \begin{cases}0, & u>x_{j} \\
\int_{u}^{x_{j}}\left(x_{j}-t\right)^{s-2} d t, & u \leq x_{j}\end{cases} \\
& =\frac{1}{(s-1) !} \sum_{j=1}^{n}\left(x_{j}-u\right)_{+}^{s-1} \ell_{j}(x) .
\end{aligned}
$$

Then from

$$
\int_{u}^{1} K_{s-1}(t) d t=\frac{1}{(s-2) !} \int_{u}^{1}(x-t)_{+}^{s-2} d t-\frac{1}{(s-2) !} \sum_{j=1}^{n} \int_{u}^{1}\left(x_{j}-t\right)_{+}^{s-2} \ell_{j}(x) d t,
$$

we get $\int_{u}^{1} K_{s-1}(t) d t=K_{s}(u)$ by $(2.7)$ and $(2.8)$.

In the following, we consider functions of limited regularities as

$$
\begin{aligned}
& \text { Suppose that } f(t) \text { has an absolutely continuous }(r-1) \text { st derivative } f^{(r-1)} \text { on }[-1,1] \\
& \text { for some } r \geq 1 \text { with } f^{(r-1)}(t)=f^{(r-1)}(-1)+\int_{-1}^{t} g(y) d y \text {, where } g \text { is absolutely } \\
& \text { integrable and of bounded variation } \operatorname{Var}(g)<\infty \text { on }[-1,1] \text {. }
\end{aligned}
$$

From Stein and Shakarchi [53, p. 130] and Tao [56, pp. 143-145], we see that a function $G:[-1,1] \rightarrow R$ is absolutely continuous if and only if it takes the form $G(t)=\int_{-1}^{t} g(y) d y+C$ for some absolutely integrable $g:[-1,1] \rightarrow R$ and a constant $C$. It is obvious that such $g$ is not unique. Then in this paper, we suppose $f(t)$ satisfies (2.9) and define

$$
V_{r}=\inf \left\{\begin{array}{l|l}
\operatorname{Var}(g) \mid \begin{array}{l}
f^{(r-1)}(t)=f^{(r-1)}(-1)+\int_{-1}^{t} g(y) d y \text { for all } t \in[-1,1] \text { with } g \text { being } \\
\text { absolutely integrable and of bounded variation }
\end{array}
\end{array}\right\}
$$

REMARK 1. Here, we use the condition " $f^{(r-1)}(t)=f^{(r-1)}(-1)+\int_{-1}^{t} g(y) d y$, where $g$ is absolutely integrable and of bounded variation $\operatorname{Var}(g)<\infty$ " instead of " $f^{(r)}$ is of bounded variation $V_{r}=\operatorname{Var}\left(f^{(r)}\right)<\infty$ " in [58, 59]. If $f^{(r)}$ is of bounded variation, then $f^{(r+1)}$ exists almost everywhere and $f^{(r+1)} \in L^{1}([-1,1])$ (see Lang [39] and Rudin [48]). Whereas, $f^{(r)}$ in [58,59] denotes an equivalent representation in the sense of almost everywhere. An example for $f(x)=|t|$ is given in [58, 59, where $f(t)$ is not differentiable at $t=0$, but $f^{\prime}$ can be chosen as

$$
f^{\prime}(t)= \begin{cases}1, & t>0 \\ c, & t=0 \\ -1, & t<0\end{cases}
$$

then $\operatorname{Var}\left(f^{\prime}\right)=\left\{\begin{array}{ll}2, & |c| \leq 1 \\ |1+c|+|1-c|, & \text { otherwise }\end{array}\right.$. Using the new condition, we see that $|t|$ can be represented as $|t|=1+\int_{-1}^{t} g(y) d y$ with $g(y)=\left\{\begin{array}{ll}1, & y>0 \\ c, & y=0 \\ -1, & y<0\end{array}\right.$ and $V_{1}=2$ is unique.

Theorem 2.2. Suppose $f(t)$ satisfies (2.9), then for $n \geq r$, we have

$$
\left\|E_{n}[f]\right\|_{\infty} \leq V_{r}\left\|K_{r+1}\right\|_{\infty} .
$$

Proof. Applying the Peano theorem implies that for each fixed $x \in[-1,1]$,

$$
E_{n}[f](x)=\int_{-1}^{1} f^{(s)}(t) K_{s}(t) d t, \quad s=1,2, \ldots, r-1 .
$$


Then, directly following Brass and Petras [6], integrating by parts and using $K_{r}(-1)=K_{r}(1)=$ 0 yields

$$
E_{n}[f](x)=\int_{-1}^{1} f^{(r-1)}(t) K_{r-1}(t) d t=\int_{-1}^{1} g(t) K_{r}(t) d t
$$

Since $g$ can be written as $g=g_{1}-g_{2}$ with $g_{1}$ and $g_{2}$ are monotonically increasing, and $\operatorname{Var}(g)=\operatorname{Var}\left(g_{1}\right)+\operatorname{Var}\left(g_{2}\right)$ (see Lang [39, pp. 280-281]). Without loss of generality, assume $g$ is monotonically increasing. Then by the second mean value theorem of integral calculus, it follows from $K_{r+1}(-1)=\int_{-1}^{1} K_{r}(t) d t=0$ that there exists a $\xi \in[-1,1]$ such that

$$
E_{n}[f](x)=g(-1) \int_{-1}^{\xi} K_{r}(t) d t+g(1) \int_{\xi}^{1} K_{r}(t) d t=(g(1)-g(-1)) K_{r+1}(\xi)=\operatorname{Var}(g) K_{r+1}(\xi)
$$

which leads to the desired result. $\square$

Lemma 2.3. [6, Lemma 5.7.1] Assume that

$$
\sup _{-1 \leq t \leq 1} w(t) \sqrt{1-t^{2}}<\infty, \quad t_{u}(y)= \begin{cases}0, & y<u \\ 1, & y \geq u\end{cases}
$$

Then, for every positive integer $\ell$ and every $u \in[-1,1]$, there is a $q_{u} \in \mathcal{P}_{\ell}$ satisfying

$$
q_{u}(y) \geq t_{u}(y) \quad \text { for all } y \in[-1,1]
$$

and

$$
\int_{-1}^{1}\left[t_{u}(y)-q_{u}(y)\right] w(y) d y \geq-\frac{\pi}{\ell+1} \sup _{-1 \leq t \leq 1} w(t) \sqrt{1-t^{2}}
$$

LEMMA 2.4.

$$
\left|K_{s+1}(u)\right| \leq \frac{\pi}{n-s+1} \sup _{-1 \leq t \leq 1}\left|K_{s}(t)\right|
$$

Proof. In Lemma 2.3, letting $\ell=n-s-1, w(t) \equiv 1$, representing $q_{u}$ as $q_{u}(t)=p_{n-1}^{(s)}(t)$, and noting that $E_{n}\left[\mathcal{P}_{n-1}\right]=0$, by Theorem 2.2 we have

$$
0=E_{n}\left[p_{n-1}\right]=\int_{-1}^{1} p_{n-1}^{(s)}(t) K_{s}(t) d t=\int_{-1}^{1} q_{u}(t) K_{s}(t) d t
$$

Consequently, by Lemma 2.3 we get that

$$
\begin{aligned}
\left|K_{s+1}(u)\right|=\left|\int_{u}^{1} K_{s}(t) d t\right|=\left|\int_{-1}^{1} K_{s}(t) t_{u}(t) d t\right| & =\left|\int_{-1}^{1} K_{s}(t)\left[t_{u}(t)-q_{u}(t)\right] d t\right| \\
& \leq \frac{\pi}{n-s} \sup _{-1 \leq t \leq 1}\left|K_{s}(t)\right|
\end{aligned}
$$

From Theorem 2.2 and Lemma 2.4 we obtain that

THEOREM 2.5. Suppose $f(t)$ satisfies (2.9), then for $n \geq r+1$

$$
\left\|E_{n}[f]\right\|_{\infty} \leq \frac{\pi^{r} V_{r}}{(n-1)(n-2) \cdots(n-r)}\left\|K_{1}\right\|_{\infty}
$$


3. Wainerman's lemma. In the following, we shall focus on the estimate of $\left\|K_{1}\right\|_{\infty}$. Notice that $\sum_{j=1}^{n} \ell_{j}(t) \equiv 1$ for $t \in[-1,1]$ and

$$
K_{1}(u)=(x-u)_{+}^{0}-\sum_{j=1}^{n}\left(x_{j}-u\right)_{+}^{0} \ell_{j}(x) .
$$

If $x_{1}<u \leq 1$, we have $K_{1}(u)=1$ for $u \leq x$, and $K_{1}(u)=0$ for $u>x$. While for $-1<u \leq x_{n}$, we have $K_{1}(u)=0$ for $u \leq x$, and $K_{1}(u)=-1$ for $u>x$. Thus, in these cases we obtain

$$
\left|K_{1}(u)\right| \leq 1 \leq \max _{1 \leq j \leq n}\left\|\ell_{j}\right\|_{\infty}
$$

since $\ell_{j}\left(x_{j}\right)=1$ for $j=1,2, \ldots, n$.

Suppose that $x_{m+1}<u \leq x_{m}$ for some positive integer $m$, then for $u \leq x$ we get

$$
K_{1}(u)=1-\sum_{j=1}^{n}\left(x_{j}-u\right)_{+}^{0} \ell_{j}(x)=1-\sum_{j=1}^{m} \ell_{j}(x)=\sum_{j=m+1}^{n} \ell_{j}(x),
$$

while for $u>x$ we have

$$
K_{1}(u)=-\sum_{j=1}^{n}\left(x_{j}-u\right)_{+}^{0} \ell_{j}(x)=-\sum_{j=1}^{m} \ell_{j}(x) .
$$

Lemma 3.1. (Wainerman's lemma [72]) Suppose $x_{m+1}<u \leq x_{m}$ for some positive integer $m$, and let

$$
a_{k}(u)= \begin{cases}\sum_{j=1}^{k} \ell_{j}(u), & k=1,2, \ldots, m \\ \sum_{j=k}^{n} \ell_{j}(u), & k=m+1, m+2, \ldots, n\end{cases}
$$

and $a_{0}(u)=a_{n+1}(u) \equiv 0$. Then it follows for $x_{m+1}<u<x_{m}$ that

$$
\operatorname{sgn}\left(a_{k}(u)\right)=\operatorname{sgn}\left(\ell_{k}(u)\right)= \begin{cases}(-1)^{m-k}, & k=1,2, \ldots, m \\ (-1)^{k-m-1}, & k=m+1, m+2, \ldots, n\end{cases}
$$

and for $x_{m+1}<u \leq x_{m}$ that

$$
\left|a_{k}(u)\right| \leq\left|\ell_{k}(u)\right|, \quad k=1,2, \ldots, n,
$$

where sgn denotes the sign function.

Proof. The interesting result and its proof is published in Russian in [72. For convenience and completeness, we present the proof here.

For $x_{m+1}<u<x_{m}$, from the definition of $\ell_{k}(t)$ we see that

$$
\begin{aligned}
\operatorname{sgn}\left(\ell_{k}(u)\right) & =\operatorname{sgn}\left(\frac{\left(u-x_{1}\right) \cdots\left(u-x_{k-1}\right)\left(u-x_{k+1}\right) \cdots\left(u-x_{n}\right)}{\left(x_{k}-x_{1}\right) \cdots\left(x_{k}-x_{k-1}\right)\left(x_{k}-x_{k+1}\right) \cdots\left(x_{k}-x_{n}\right)}\right) \\
& =(-1)^{1-k} \operatorname{sgn}\left(\left(u-x_{1}\right) \cdots\left(u-x_{k-1}\right)\left(u-x_{k+1}\right) \cdots\left(u-x_{n}\right)\right),
\end{aligned}
$$

which directly leads to the desired result (3.5) for $\operatorname{sgn}\left(\ell_{k}(u)\right)$ based on $k \leq m$ or $k>m$, respectively.

In the following, we will show that $\operatorname{sgn}\left(a_{k}(u)\right)$ also satisfies (3.5).

In the case $k \leq m$ : Since

$$
a_{k}\left(x_{j}\right)=\sum_{i=1}^{k} \ell_{i}\left(x_{j}\right)=\left\{\begin{array}{ll}
1, & j=1,2, \ldots, k \\
0, & j=k+1, k+2, \ldots, n
\end{array},\right.
$$

then by the Rolle's theorem it follows

$$
a_{k}^{\prime}\left(y_{j}\right)=0
$$

for some $y_{j}$ satisfying $x_{j+1}<y_{j}<x_{j}$ for $j=1, \ldots, k-1, k+1, \ldots, n-1$. 


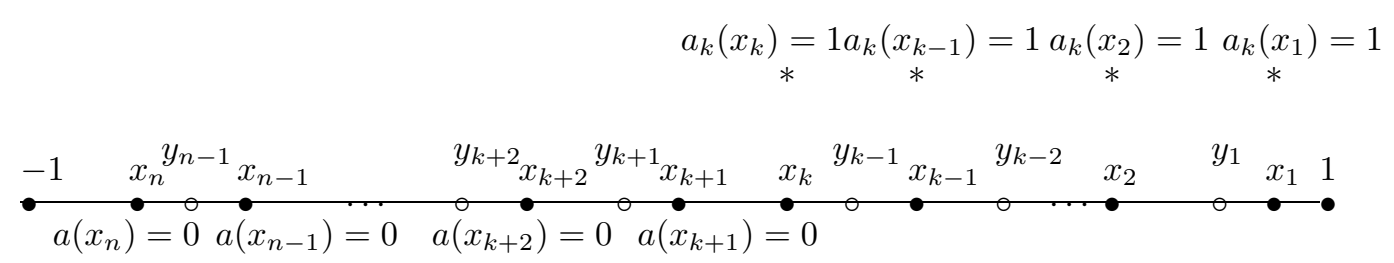

Note that $a_{k}(t)$ is a polynomial of degree $n-1$, then $a_{k}^{\prime}(t)$ is a polynomial of degree $n-2$, which implies that $y_{j}$ are the exact zeros of $a_{k}^{\prime}(t)$ and then $a_{k}^{\prime}(t)$ has the form of

$$
a_{k}^{\prime}(t)=C\left(t-y_{1}\right) \cdots\left(t-y_{k-1}\right)\left(t-y_{k+1}\right) \cdots\left(t-y_{n-1}\right)
$$

for some non-zero constant $C$. In addition, from (3.7) $a_{k}^{\prime}(t)$ has alternative sign between these roots. Then, by $a_{k}\left(x_{k+1}\right)=0$ and $a_{k}\left(x_{k}\right)=1$, it yields

$$
a_{k}^{\prime}(t)>0, \quad t \in\left(y_{k+1}, y_{k-1}\right)
$$

and

$$
\operatorname{sgn}\left(a_{k}(t)\right)=1, \quad t \in\left(x_{k+1}, x_{k}\right) \subset\left(y_{k+1}, y_{k-1}\right)
$$

since $a(t)$ is strictly increasing in $\left(x_{k+1}, x_{k}\right)$ and $a_{k}\left(x_{k+1}\right)=0$.

By the alternative property of $a_{k}^{\prime}(t)$ between these roots, it deduces that $\operatorname{sgn}\left(a_{k}^{\prime}(t)\right)=$ $(-1)^{j-k}$ for $t \in\left(y_{j+1}, y_{j}\right)$ and $j>k$, particularly,

$$
\operatorname{sgn}\left(a_{k}^{\prime}(t)\right)=1, \quad t \in\left(y_{k+1}, x_{k+1}\right) \subset\left(y_{k+1}, y_{k-1}\right)
$$

and

$$
\operatorname{sgn}\left(a_{k}^{\prime}(t)\right)=-1, \quad t \in\left(x_{k+2}, y_{k+1}\right) \subset\left(y_{k+2}, y_{k+1}\right),
$$

which, together with $a_{k}\left(x_{k+1}\right)=a_{k}\left(x_{k+2}\right)=0$, derives $\operatorname{sgn}\left(a_{k}(t)\right)=-1$ for $t \in\left(x_{k+2}, x_{k+1}\right)$. Similarly, applying

$$
\operatorname{sgn}\left(a_{k}^{\prime}(t)\right)=(-1)^{j-k}, \quad t \in\left(y_{j+1}, x_{j+1}\right) ; \quad \operatorname{sgn}\left(a_{k}^{\prime}(t)\right)=(-1)^{j-k+1}, \quad t \in\left(x_{j+2}, y_{j+1}\right),
$$

together with $a_{k}\left(x_{j+2}\right)=a_{k}\left(x_{j+1}\right)=0$ for $j>k$, derives $\operatorname{sgn}\left(a_{k}(t)\right)=(-1)^{j-k+1}$ for $j>k$ and $t \in\left(x_{j+2}, x_{j+1}\right)$ by induction. So we get $a_{k}(u)=(-1)^{m-k}$.

In the case $k>m$ : By

$$
a_{k}\left(x_{j}\right)=\sum_{i=k}^{n} \ell_{i}\left(x_{j}\right)=\left\{\begin{array}{ll}
0, & j=1,2, \ldots, k-1 \\
1, & j=k, k+1, \ldots, n
\end{array},\right.
$$

applying similar arguments derives $a_{k}(u)=(-1)^{k-m-1}$ for $k>m$.

Furthermore, from (3.5) and the definition of $a_{k}(t)$, we see that immediately: for $k \leq m$ and $x_{m+1}<u<x_{m}$,

$$
\left|a_{k}(u)\right|=\left|\ell_{k}(u)+a_{k-1}(u)\right|=\left|\ell_{k}(u)\right|-\left|a_{k-1}(u)\right| \leq\left|\ell_{k}(u)\right|,
$$

and for $k>m$

$$
\left|a_{k}(u)\right|=\left|\ell_{k}(u)+a_{k+1}(u)\right|=\left|\ell_{k}(u)\right|-\left|a_{k+1}(u)\right| \leq\left|\ell_{k}(u)\right| .
$$

The special case of (3.6) for $u=x_{m}$ directly follows from $\left|a_{k}\left(x_{m}\right)\right|=\left|\ell_{k}\left(x_{m}\right)\right|$ by the definitions of $a_{k}(u)$ and $\ell_{k}(u)$.

Theorem 2.5 together with (3.2), (3.3), (3.4) and (3.6) leads to the following estimate.

TheOrem 3.2. Suppose $f(t)$ satisfies (2.9), then for $n \geq r+1$

$$
\left\|E_{n}[f]\right\|_{\infty} \leq \frac{\pi^{r} V_{r}}{(n-1)(n-2) \cdots(n-r)} \max _{1 \leq j \leq n}\left\|\ell_{j}\right\|_{\infty}
$$

In the next section, we shall focus on estimates of $\max _{1 \leq j \leq n}\left\|\ell_{j}\right\|_{\infty}$ for special points of sets. 
4. Estimates of $\left\|\ell_{j}\right\|_{\infty}$ and convergence rates on $\left\|f-L_{n}[f]\right\|_{\infty}$. For any convergent quadrature derived from polynomial interpolation at the grid points (1.1) for

$$
\int_{-1}^{1} f(x) w(x) d x=\int_{-1}^{1} f(x) d \sigma(x)
$$

for each $\sigma(x)$ of bounded variation and any analytic function $f(x)$ on $[-1,1]$, the clustering of the $n$ points has a limiting Chebyshev distribution

$$
\mu(t)=\frac{1}{\pi} \int_{-1}^{t} \frac{1}{\sqrt{1-x^{2}}} d x
$$

(see Krylov [37, Theorem 7, p. 263]); that is, the clustering will be asymptotically the same: on $[-1,1], n$ points will be distributed with density

$$
\frac{n}{\pi \sqrt{1-x^{2}}}
$$

as $n$ tends to infinity (see Hale and Trefethen [27] and Trefethen [58]).

Moreover, the clustering of optimal pointsystems for polynomial interpolation implies near endpoints \pm 1 (see Z. Ditzian and V. Totik [13] and [58]). (The Gauss-Jacobi type pointsystems have this proposition.) The density of the zeros of orthogonal polynomials has been extensively studied in Erdös and Turán [16, 17], Gatteschi [22] and Szegö [55].

4.1. Strongly normal pointsystems. One of the proofs of Weierstrass approximation theorem using interpolation polynomials was first presented by Fejér [19 in 1916 based on the Chebyshev pointsystem of first kind $\left\{x_{k}=\cos \left(\frac{2 k-1}{2 n} \pi\right)\right\}_{k=1}^{n}$ : If $f \in C([-1,1])$, then there is a unique polynomial $H_{2 n-1}(f, t)$ of degree at most $2 n-1$ such that $\lim _{n \rightarrow \infty}\left\|H_{2 n-1}(f)-f\right\|_{\infty}=0$, where $H_{2 n-1}(f, t)$ is determined by

$$
H_{2 n-1}\left(f, x_{k}\right)=f\left(x_{k}\right), \quad H_{2 n-1}^{\prime}\left(f, x_{k}\right)=0, \quad k=1,2, \ldots, n .
$$

This polynomial is known as the Hermite-Fejér interpolation polynomial.

The convergence result has been extended to general Hermite-Fejér interpolation of $f(x)$ at nodes (1.1) by Grünwald [25] in 1942, upon strongly normal pointsystems introduced in Fejér 20]: Given, respectively, the function values $f\left(x_{1}\right), f\left(x_{2}\right), \ldots, f\left(x_{n}\right)$ and derivatives $d_{1}, d_{2}, \ldots$, $d_{n}$ at these grids, the general Hermite-Fejér interpolation polynomial $H_{2 n-1}(f)$ has the form of

$$
H_{2 n-1}(f, t)=\sum_{k=1}^{n} f\left(x_{k}\right) h_{k}(t)+\sum_{k=1}^{n} d_{k} b_{k}(t)
$$

where $h_{k}(t)=v_{k}(t)\left(\ell_{k}(t)\right)^{2}, b_{k}(t)=\left(t-x_{k}\right)\left(\ell_{k}(t)\right)^{2}$ and

$$
v_{k}(t)=1-\left(t-x_{k}\right) \frac{\omega_{n}^{\prime \prime}\left(x_{k}\right)}{\omega_{n}^{\prime}\left(x_{k}\right)} \quad(\text { see Fejér [21]). }
$$

The pointsystem (1.1) is called strongly normal if for all $n$

$$
v_{k}(t) \geq c>0, \quad k=1,2, \ldots, n, \quad t \in[-1,1]
$$

for some positive constant $c$. The pointsystem (1.1) is called normal if for all $n$

$$
v_{k}(t) \geq 0, \quad k=1,2, \ldots, n, \quad t \in[-1,1] .
$$

Fejér [20] (also see Szegö [55, p. 339]) showed that for the zeros of Jacobi polynomial $P_{n}^{(\alpha, \beta)}(t)$ of degree $n(\alpha>-1, \beta>-1)$

$$
v_{k}(t) \geq \min \{-\alpha,-\beta\} \text { for }-1<\alpha \leq 0,-1<\beta \leq 0, k=1,2, \ldots, n \text { and } t \in[-1,1] .
$$


While for the Legendre-Gauss-Lobatto pointsystem (the roots of $\left.\left(1-t^{2}\right) P_{n-2}^{(1,1)}(t)=0\right)$,

$$
v_{k}(t) \geq 1, \quad k=1,2, \ldots, n, \quad t \in[-1,1] \text { (Fejér [21]). }
$$

These results have been extended to Jacobi-Gauss-Lobatto pointsystem (the roots of (1$\left.\left.t^{2}\right) P_{n-2}^{(\alpha, \beta)}(t)=0\right)$ and Jacobi-Gauss-Radau pointsystem (the roots of $(1-t) P_{n-1}^{(\alpha, \beta)}(t)=0$ or $\left.(1+t) P_{n-1}^{(\alpha, \beta)}(t)=0\right)$ by Vértesi [64, 65]: for all $k$ and $t \in[-1,1]$,

$$
\begin{array}{cc}
v_{k}(t) \geq \min \{2-\alpha, 2-\beta\} & \text { for }\left\{x_{k}\right\} \bigcup\{-1,1\} \text { with } 1 \leq \alpha \leq 2 \text { and } 1 \leq \beta \leq 2, \\
v_{k}(t) \geq \min \{2-\alpha,-\beta\} & \text { for }\left\{x_{k}\right\} \bigcup\{1\} \text { with } 1 \leq \alpha \leq 2 \text { and }-1<\beta \leq 0, \\
v_{k}(t) \geq \min \{-\alpha, 2-\beta\} & \text { for }\left\{x_{k}\right\} \bigcup\{-1\} \text { with }-1<\alpha \leq 0 \text { and } 1 \leq \beta \leq 2 .
\end{array}
$$

Proposition 4.1. (i) [20, 55] The Gauss-Jacobi pointsystem is strongly normal if and only if $\max \{\alpha, \beta\}<0$.

(ii) 64, 65] The Jacobi-Gauss-Lobatto pointsystem is strongly normal if and only if $1 \leq \alpha<$ 2 and $1 \leq \beta<2$.

(iii) 64, 65] The Jacobi-Gauss-Radau pointsystem including $x_{1}=1$ is strongly normal if and only if $1 \leq \alpha<2$ and $-1 \leq \beta<0$, and the Jacobi-Gauss-Radau pointsystem including $x_{n}=-1$ is strongly normal if and only if $-1<\alpha<0$ and $1 \leq \beta<2$.

It is worth noticing that if the pointsystem is strongly normal, then it implies $v_{i}(t) \geq c>0$ for all $i=1,2, \ldots, n$ and $t \in[-1,1]$, and

$$
1 \equiv \sum_{i=1}^{n} h_{i}(t)=\sum_{i=1}^{n} v_{i}(t) \ell_{i}^{2}(t) \geq c \sum_{i=1}^{n} \ell_{i}^{2}(t)
$$

(see [20]) and then

$$
\left\|\ell_{i}\right\|_{\infty} \leq \frac{1}{\sqrt{c}}, \quad i=1,2, \ldots, n .
$$

TheOREM 4.2. Suppose $f(t)$ satisfies (2.9) and $\left\{x_{j}\right\}_{j=1}^{n}$ is a strongly normal pointsystem, then for $n \geq r+1$

$$
\left\|E_{n}[f]\right\|_{\infty} \leq \frac{\pi^{r} V_{r}}{\sqrt{c}(n-1)(n-2) \cdots(n-r)} .
$$

Following de la Vallée Poussin $\underline{62}$, the error bound indicates that $\left\|f-L_{n}[f]\right\|_{\infty}$ has the same asymptotic order as the estimate of $\left\|f-p_{n-1}^{*}\right\|_{\infty}$ for the interpolant at a strongly normal pointsystem for a functions of limited regularity with $V_{r}<\infty$ for some $r \geq 1$.

To check the error bounds in Theorem 4.2 numerically, we consider two limited regularity functions: $f(x)=|x|\left(V_{1}<\infty\right)$ and $f(x)=|x|^{3}\left(V_{3}<\infty\right)$. All $(\alpha, \beta)$ are generated by rand $(1,2)$ 2 except for $(\alpha, \beta)=(-0.5,-0.5),(\alpha, \beta)=(0,0),(\alpha, \beta)=(1,1)$ or $(\alpha, \beta)=(1.5,1.5)$. Particularly, we used $-\operatorname{rand}(1,2)$ in Figs. 4.1-4.2 for strongly normal Gauss-Jacobi pointsystems, while $\operatorname{rand}(1,2)+1$ in Figs. 4.3-4.4 for strongly normal Jacobi-Gauss-Lobatto pointsystems. In Figs. 4.5-4.6, we used $(\operatorname{rand}(1)+1,-\operatorname{rand}(1))(1$ st row) and $(-\operatorname{rand}(1), \operatorname{rand}(1)+1)(2$ nd row) for strongly normal Jacobi-Gauss-Radau pointsystems, respectively.

From Figs. 4.1-4.6, we see that these convergence rates are in conformity to the estimates and attainable.

\footnotetext{
${ }^{2} \operatorname{rand}(\mathrm{m}, \mathrm{n})$ returns an $\mathrm{m}-\mathrm{by}-\mathrm{n}$ matrix containing pseudorandom values drawn from the standard uniform distribution on the open interval $(0,1)$.
} 

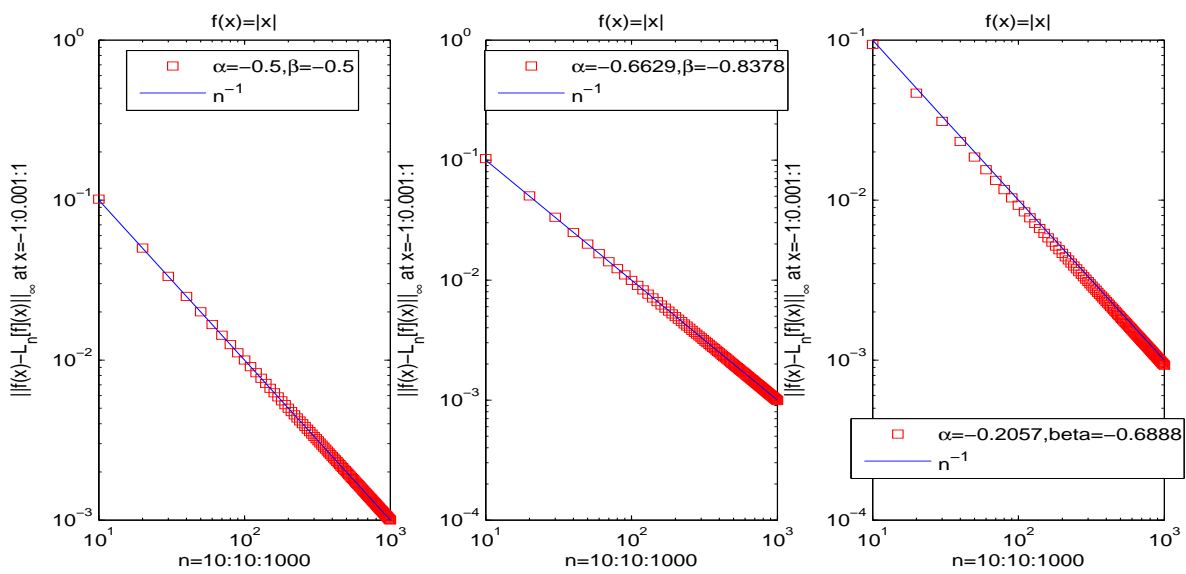

FIG. 4.1. $\max _{x=-1: 0.001: 1}\left|f(x)-L_{n}[f](x)\right|$ with $n=10: 10: 1000$ at the strongly normal Gauss-Jacobi pointsystems for $f(x)=|x|$, respectively.
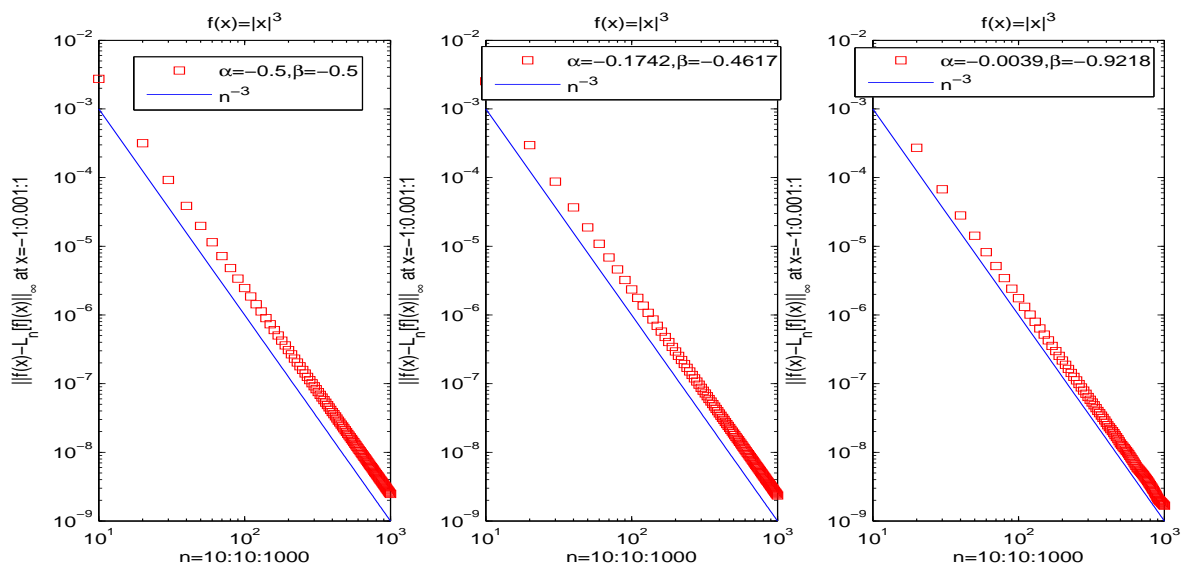

FIG. 4.2. $\max _{x=-1: 0.001: 1}\left|f(x)-L_{n}[f](x)\right|$ with $n=10: 10: 1000$ at the strongly normal Gauss-Jacobi pointsystems for $f(x)=|x|^{3}$, respectively.
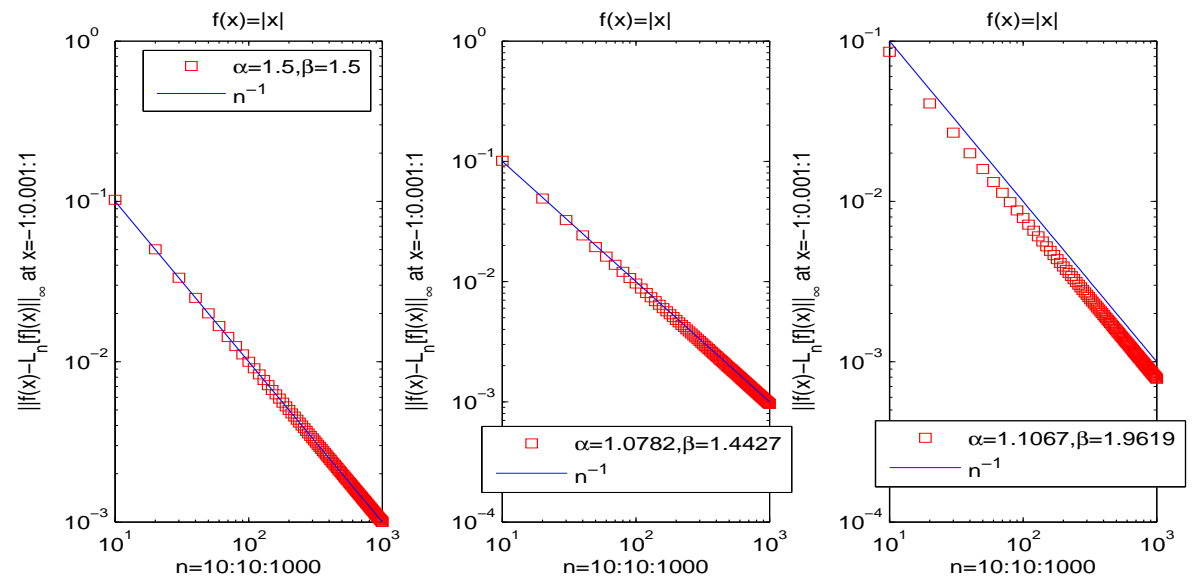

FIG. 4.3. $\max _{x=-1: 0.001: 1}\left|f(x)-L_{n}[f](x)\right|$ with $n=10: 10: 1000$ at the strongly normal Jacobi-GaussLobatto pointsystems for $f(x)=|x|$, respectively. 

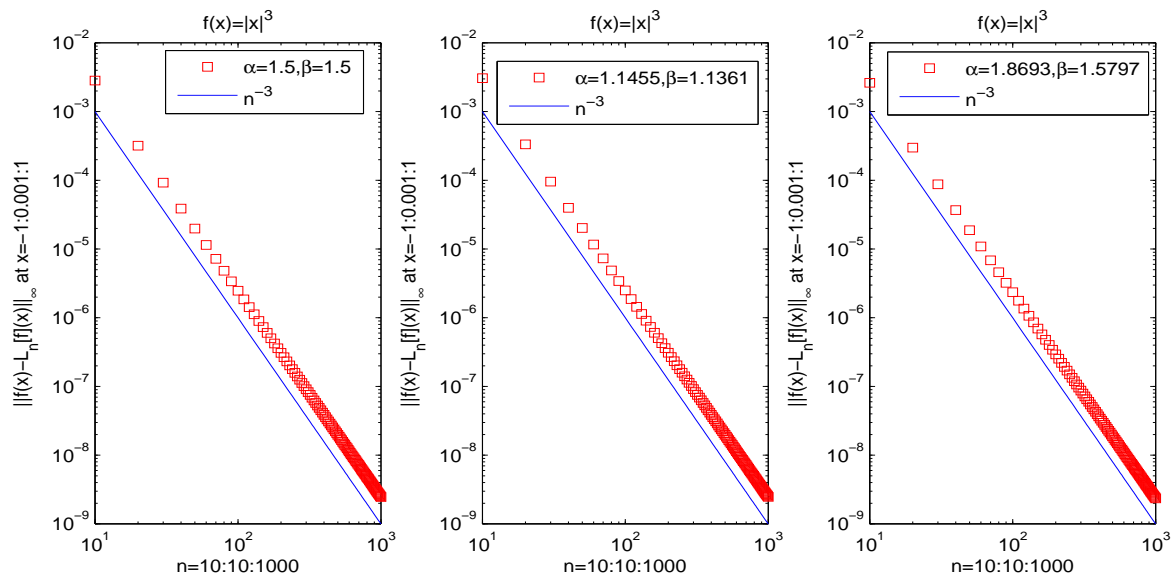

FIG. 4.4. $\max _{x=-1: 0.001: 1}\left|f(x)-L_{n}[f](x)\right|$ with $n=10: 10: 1000$ at the strongly normal Jacobi-GaussLobatto pointsystems for $f(x)=|x|^{3}$, respectively.
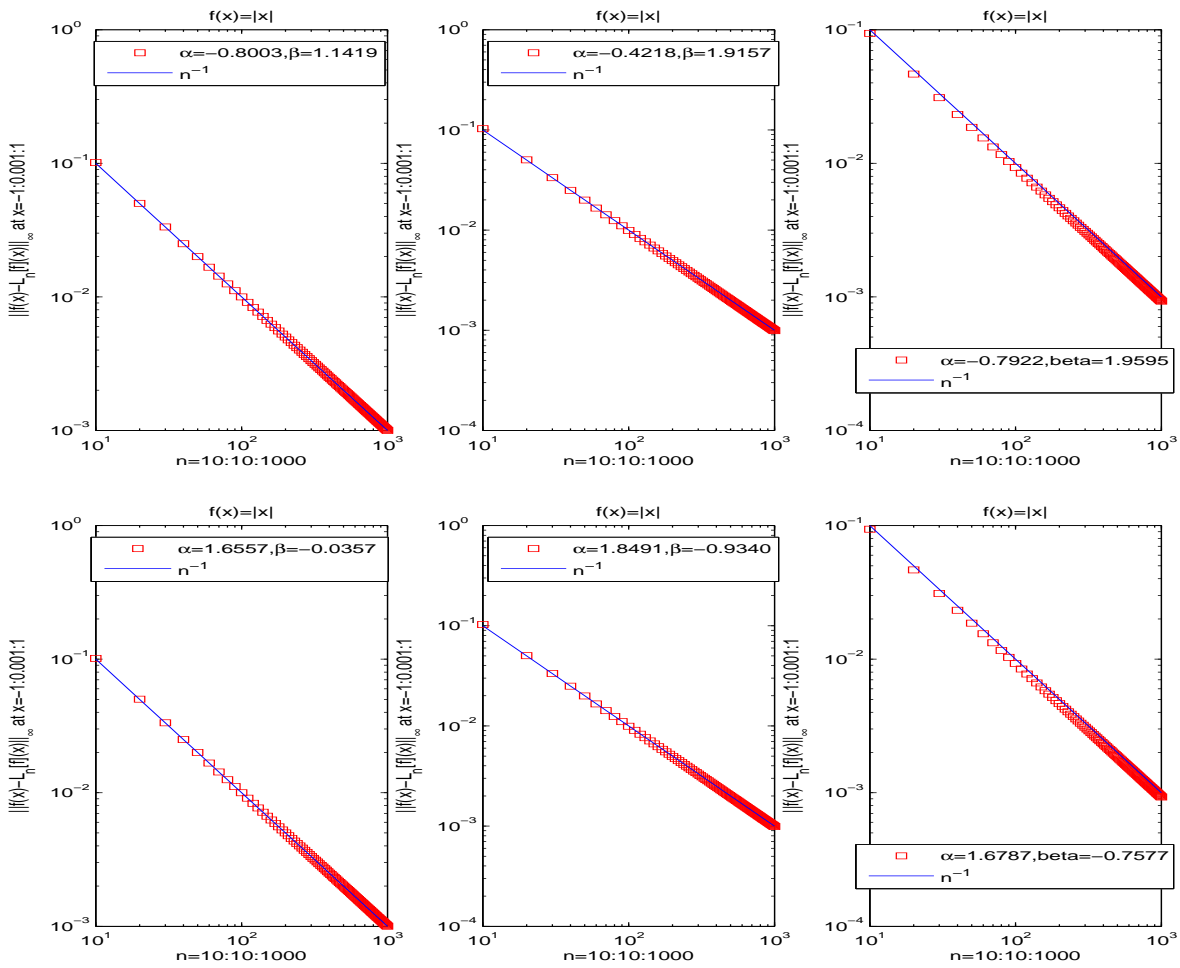

FIG. 4.5. $\max _{x=-1: 0.001: 1}\left|f(x)-L_{n}[f](x)\right|$ with $n=10: 10: 1000$ at the strongly normal Jacobi-GaussRadau pointsystems including -1 (1st row) and 1 (2nd row) for $f(x)=|x|$, respectively.

4.2. General Gauss-Jacobi pointsystems. In this subsection, we will consider convergence rates for general Gauss-Jacobi pointsystems, which includes the corresponding strongly normal pointsystems $(-1<\alpha, \beta<0)$ as special cases.

Let $\left\{x_{k}\right\}_{k=1}^{n}$ be the roots of the Jacobi polynomial $P_{n}^{(\alpha, \beta)}(t)(\alpha, \beta>-1)$ and $x_{k}=\cos \left(\theta_{k}\right)$. Then from Szegö [55], it follows

$$
\left.P_{n}^{(\alpha, \beta)}(t)=(-1)^{n} P_{n}^{(\beta, \alpha)}(-t) \quad(\underline{55},(4.1 .3)]\right)
$$



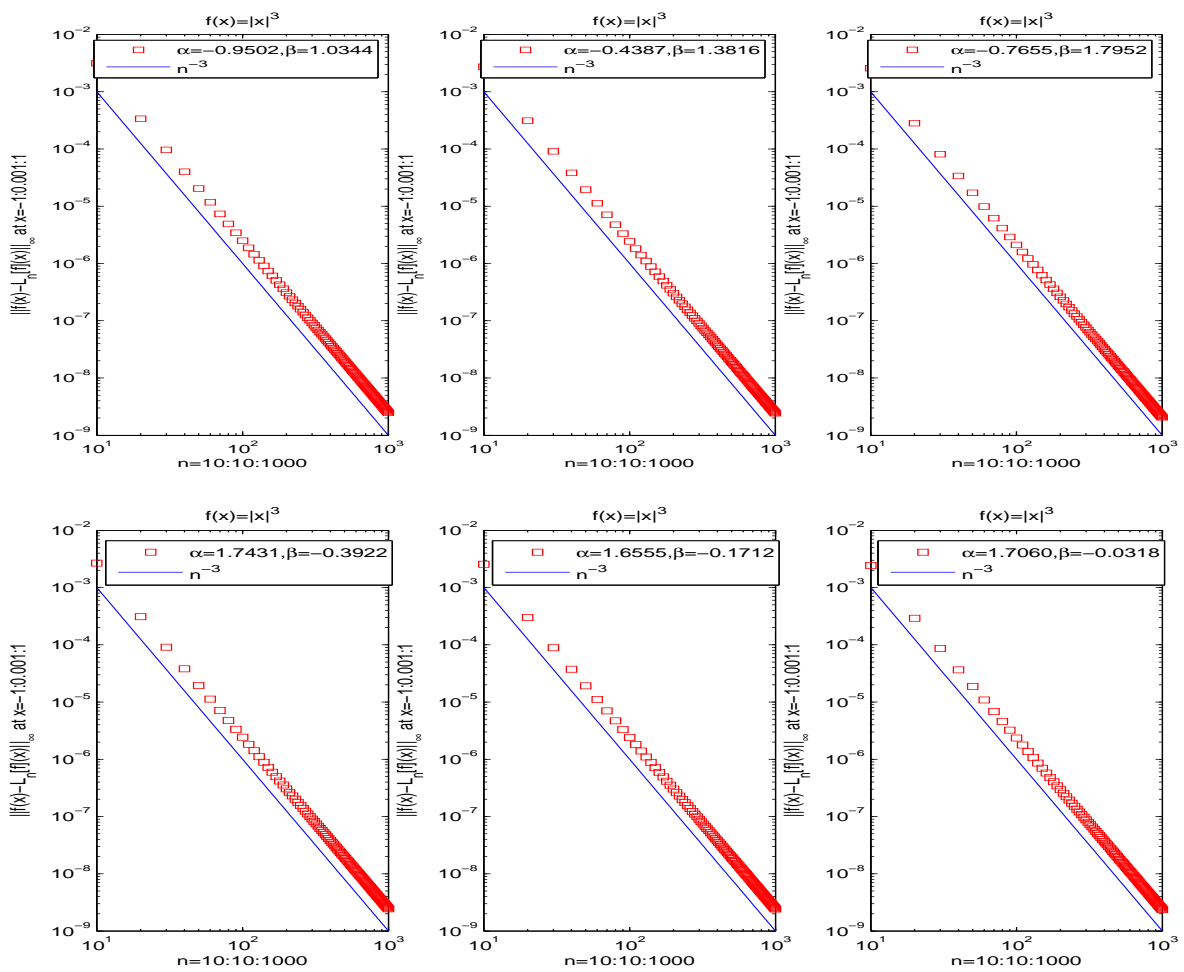

FIG. 4.6. $\max _{x=-1: 0.001: 1}\left|f(x)-L_{n}[f](x)\right|$ with $n=10: 10: 1000$ at the strongly normal Jacobi-GaussRadau pointsystems including -1 (1st row) and 1 (2nd row) for $f(x)=|x|^{3}$, respectively.

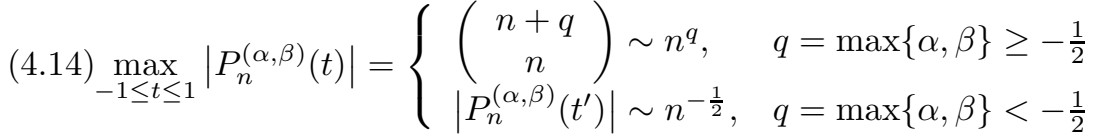

where $x^{\prime}$ is one of the two maximum points, and for $t=\cos (\theta)$ and any fixed constant $c$ with $0<c<1$

$$
\begin{aligned}
& P_{n}^{(\alpha, \beta)}(\cos (\theta))=\left\{\begin{array}{ll}
O\left(n^{\alpha}\right), & 0 \leq \theta \leq c n^{-1} \\
\theta^{-\alpha-\frac{1}{2}} O\left(n^{-\frac{1}{2}}\right), & c n^{-1} \leq \theta \leq \frac{\pi}{2}
\end{array} \quad(\text { [55, Theorem 7.32.2]), }\right. \\
& \theta_{k}=n^{-1}[k \pi+O(1)] \quad([55,(8.9 .1)]), \\
& \left|P_{n}^{(\alpha, \beta)^{\prime}}\left(\cos \left(\theta_{k}\right)\right)\right| \sim k^{-\alpha-\frac{3}{2}} n^{\alpha+2}, \quad 0<\theta_{k} \leq \frac{\pi}{2} \quad([55,(8.9 .2)]) .
\end{aligned}
$$$$
\text { Moreover, expression (4.17) can be extended to }
$$

$$
\left|P_{n}^{(\alpha, \beta)^{\prime}}\left(\cos \left(\theta_{k}\right)\right)\right| \sim k^{-\alpha-\frac{3}{2}} n^{\alpha+2}, 0<\theta_{k} \leq c_{1} \pi
$$

for any fixed $c_{1}$ with $0<c_{1}<1([67,(4.6)])$.

Based on these identities, the estimates on $\ell_{k}(t)=\frac{P_{n}^{(\alpha, \beta)}(t)}{P_{n}^{(\alpha, \beta)^{\prime}}\left(x_{k}\right)\left(t-x_{k}\right)}$ have been extensively studied in Kelzon [34, 35, Vértesi 66, 68, Sun [54, Prestin [46], Kvernadze [38, Vecchia et al. 69, etc.

Lemma 4.3. [54] (also see [38]) For $t \in[-1,1]$, let $x_{m}$ be the root of the Jacobi polynomial 
$P_{n}^{(\alpha, \beta)}$ which is closest to $t$. Then we have

$$
\ell_{k}(t)=\left\{\begin{array}{ll}
O\left(|k-m|^{-1}+|k-m|^{\gamma-\frac{1}{2}}\right), & k \neq m \\
O(1) & k=m
\end{array}, \quad \gamma=\max \{\alpha, \beta\},\right.
$$

for $k=1,2, \ldots, n$.

Proof. In [54, the proof of Lemma 4.3 is given only for $0 \leq \theta_{k} \leq \frac{\pi}{2}$ or $k=m$. That proof can be readily extended to $0 \leq \theta_{k} \leq \frac{2 \pi}{3}$ due to (4.18). We complement the proof for $\frac{2 \pi}{3}<\theta_{k}<\pi$ and $k \neq m$ next.

From (4.13) and (4.18), we see that

$$
\left.\left|P_{n}^{(\alpha, \beta)^{\prime}}\left(\cos \left(\theta_{k}\right)\right)\right| \sim(n-k+1)^{-\beta-\frac{3}{2}} n^{\beta+2}, \quad \frac{2 \pi}{3}<\theta_{k}<\pi \quad(\underline{46},(9)]\right) .
$$

Then for $0 \leq t=\cos (\theta) \leq 1$ with $0 \leq \theta \leq c n^{-1}$ and $\frac{2 \pi}{3}<\theta_{k}<\pi$, it follows by (4.15) and (4.20) that

$$
\ell_{k}(t)=O\left(\frac{n^{\alpha}}{(n-k+1)^{-\beta-\frac{3}{2}} n^{\beta+2}}\right)=O\left(\frac{(n-k+1)^{\beta+\frac{3}{2}}}{n^{\beta+2-\alpha}}\right)=O\left(n^{\alpha-\frac{1}{2}}\right) .
$$

While for $c n^{-1} \leq \theta \leq \frac{\pi}{2}$ and $\frac{2 \pi}{3}<\theta_{k}<\pi$, it follows by (4.15)-(4.18) and (4.20) that

$$
\ell_{k}(t)=O\left(\frac{(m \pi / n)^{-\alpha-\frac{1}{2}} n^{-\frac{1}{2}}}{(n-k+1)^{-\beta-\frac{3}{2}} n^{\beta+2}}\right)=O\left(\frac{1}{m^{\frac{1}{2}+\alpha} n^{\frac{1}{2}-\alpha}}\right)= \begin{cases}O\left(n^{\alpha-\frac{1}{2}}\right), & \alpha>-\frac{1}{2} \\ O\left(n^{-1}\right), & -1<\alpha \leq-\frac{1}{2} .\end{cases}
$$

Thus for $0 \leq t \leq 1$, we have $\ell_{k}(t)=O\left(n^{-1}+n^{\alpha-\frac{1}{2}}\right)$ for $k \neq m$, which leads to the desired result due to that $k-m \sim n$ in the case $\frac{2 \pi}{3}<\theta_{k}<\pi$.

Similarly, by (4.13) together with the above analysis, we get for $-1 \leq t \leq 0$ that

$$
\ell_{k}(t)=O\left(|k-m|^{-1}+|k-m|^{\beta-\frac{1}{2}}\right), \quad k \neq m .
$$

These together lead to the desired result (4.19) for $k \neq m$. $\square$

TheOrem 4.4. Suppose $f(t)$ satisfies (2.9) and $\left\{x_{j}\right\}_{j=1}^{n}$ are the roots of the Jacobi polynomial $P_{n}^{(\alpha, \beta)}(t)$, then for $n \geq r+1$

$$
\left\|E_{n}[f]\right\|_{\infty}=O\left(n^{-r+\max \left\{0, \gamma-\frac{1}{2}\right\}}\right), \quad \gamma=\max \{\alpha, \beta\}
$$

Proof. From Lemma 4.3, we see that $\max _{1 \leq j \leq n}\left\|\ell_{j}\right\|_{\infty}=O\left(n^{\max \left\{0, \gamma-\frac{1}{2}\right\}}\right)$, which together with Theorem 3.2 yields the desired result.

REMARK 2. Theorem 4.4 implies that $\left\|f-L_{n}[f]\right\|_{\infty}$ has the same asymptotic order as $\left\|f-p_{n-1}^{*}\right\|_{\infty}\left[62\right.$ at the roots of the Jacobi polynomial $P_{n}^{(\alpha, \beta)}(t)$ for $-1<\alpha, \beta \leq \frac{1}{2}$. Then the interpolations at the $n$-point Gauss-Legendre points and at the $n$-point Chebyshev points of first kind or second kind have essentially the same accuracy. All of them can achieve the optimal convergence rate $O\left(\left\|f-p_{n-1}^{*}\right\|_{\infty}\right)$. Consequently, the corresponding quadrature Gauss, Clenshaw-Curtis and Fejér first rule have essentially the same accuracy [74].

Here, we used Figs. 4.7-4.8 to illustrate the convergence rates for general Gauss-Jacobi pointsystems, where $(\alpha, \beta)$ are obtained by $\operatorname{rand}(1,2)\left(1\right.$ st row) and $\operatorname{mrand}(1,2)$ with $m\|\operatorname{rand}(1,2)\|_{\infty}$ $>m-1$ for $m=2,3,4$ (2nd row), respectively. From these figures, we see that the convergence rates are attainable too, which are in accordance with the estimates. Then the convergence rates at the Gauss-Jacobi pointsystems are optimal.

REMARK 3. It is of particular relevance from Figs. 4.7-4.8 in the cases that the polynomial interpolations are divergent if $r-\max \left\{0, \gamma-\frac{1}{2}\right\} \leq 0$, the divergence rate is also controlled by the order $O\left(n^{-r+\max \left\{0, \gamma-\frac{1}{2}\right\}}\right)$. 

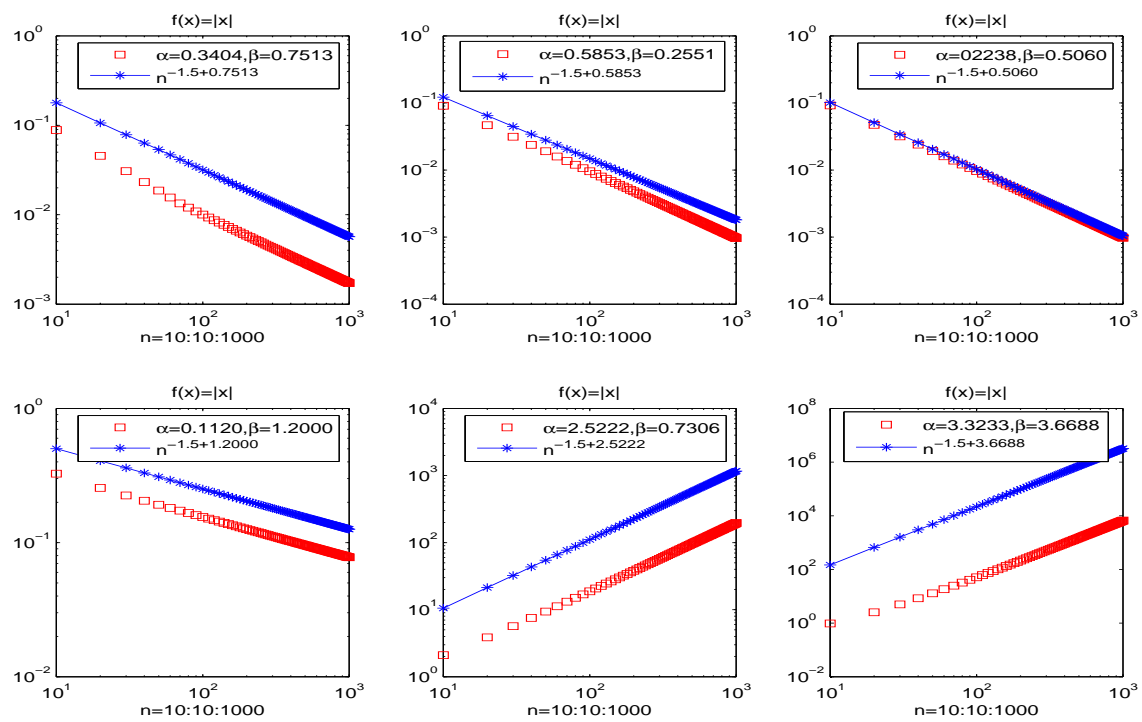

FIG. 4.7. $\max _{x=-1: 0.001: 1}\left|f(x)-L_{n}[f](x)\right|$ with $n=10: 10: 1000$ at the Gauss-Jacobi pointsystems for $f(x)=|x|$, respectively.
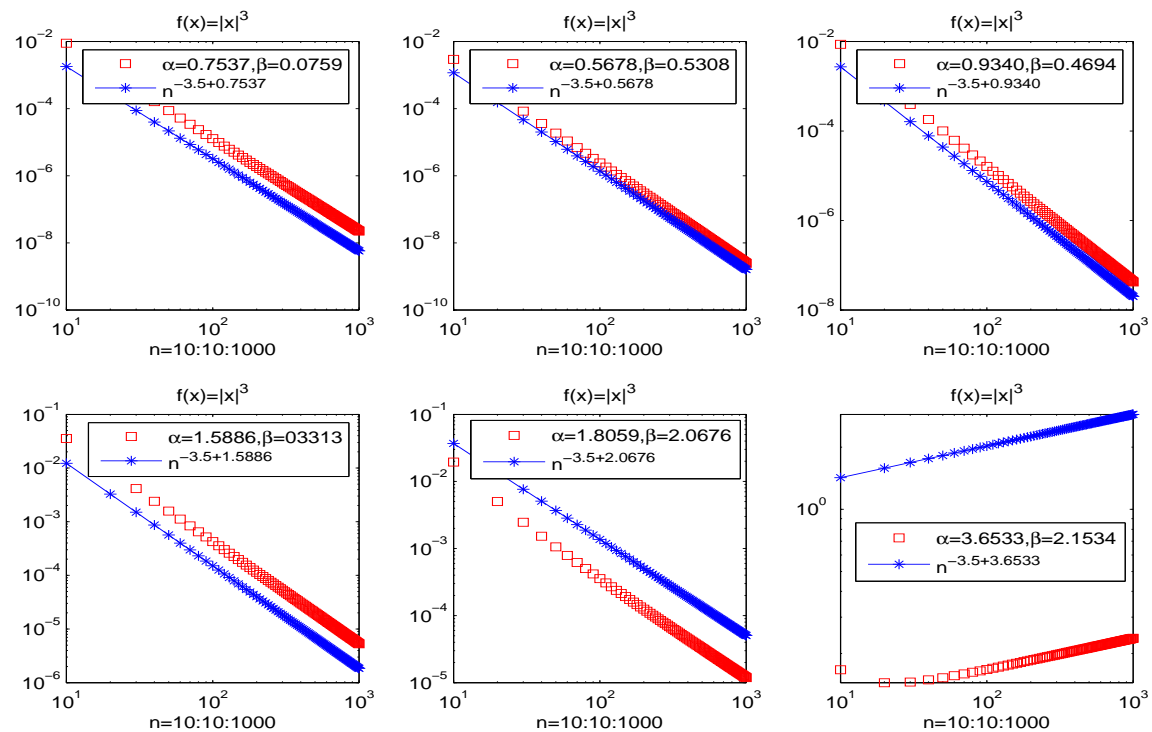

FIG. 4.8. $\max _{x=-1: 0.001: 1}\left|f(x)-L_{n}[f](x)\right|$ with $n=10: 10: 1000$ at the Gauss-Jacobi pointsystems for $f(x)=|x|^{3}$, respectively.

\subsection{General Jacobi-Gauss-Lobatto pointsystems. Let}

$$
-1=x_{n+1}<x_{n}<x_{n-1}<\cdots<x_{2}<x_{1}<x_{0}=1
$$

be the roots of $\left(1-t^{2}\right) P_{n}^{(\alpha, \beta)}(t)=0(\alpha, \beta>-1), x_{k}=\cos \left(\theta_{k}\right)$ and

$$
\omega(t)=\left(t-x_{0}\right)\left(t-x_{1}\right) \cdots\left(t-x_{n}\right)\left(t-x_{n+1}\right), \quad \ell_{k}(t)=\frac{\omega(t)}{\left(t-x_{k}\right) \omega^{\prime}\left(x_{k}\right)} .
$$

Then

$$
\ell_{0}(t)=\frac{1+t}{2} \cdot \frac{P_{n}^{(\alpha, \beta)}(t)}{P_{n}^{(\alpha, \beta)}(1)}, \quad \ell_{n+1}(t)=\frac{1-t}{2} \cdot \frac{P_{n}^{(\alpha, \beta)}(t)}{P_{n}^{(\alpha, \beta)}(-1)}
$$


and

$$
\ell_{k}(t)=\frac{\left(1-t^{2}\right) P_{n}^{(\alpha, \beta)}(t)}{\left(t-x_{k}\right)\left(1-x_{k}^{2}\right) P_{n}^{(\alpha, \beta)^{\prime}}\left(x_{k}\right)}, \quad k=1,2, \ldots, n .
$$

In the next, we shall concentrate on estimates of $\ell_{k}(t)$ for $k=0,1,2, \ldots, n+1$.

- On the estimate of $\ell_{0}(t)$ : (i) In the case $0 \leq t \leq 1$, setting $t=\cos \theta$ for $0 \leq \theta \leq \frac{\pi}{2}$, and using

$$
P_{n}^{(\alpha, \beta)}(1)=\left(\begin{array}{c}
n+\alpha \\
n
\end{array}\right) \sim n^{\alpha}([55,(4.1 .1),(7.32 .2)])
$$

we find that from (4.15) and (4.23) for $0 \leq \theta \leq \frac{\pi}{2}$,

$$
\ell_{0}(t)=\left\{\begin{array}{ll}
O(1), & 0 \leq \theta \leq c n^{-1} \\
O\left(\theta^{-\alpha-\frac{1}{2}} n^{-\frac{1}{2}} n^{-\alpha}\right)=O\left((n \theta)^{-\alpha-\frac{1}{2}}\right)=O\left(n^{-\min \left\{0, \alpha+\frac{1}{2}\right\}}\right), & c n^{-1} \leq \theta \leq \frac{\pi}{2}
\end{array} .\right.
$$

(ii) In the case $-1 \leq t \leq 0$, letting $t=-\cos (\theta)$ for $0 \leq \theta \leq \frac{\pi}{2}$ and applying $P_{n}^{(\alpha, \beta)}(-\cos (\theta))=(-1)^{n} P_{n}^{(\beta, \alpha)}(\cos (\theta))$ and $1-\cos (\theta)=2 \sin ^{2}\left(\frac{\theta}{2}\right)$ and $\frac{2}{\pi}(\theta) \leq \sin (\theta) \leq$ $\theta$, together with (4.15) and (4.23), we have

$$
\begin{aligned}
\ell_{0}(t) & =O\left(\theta^{2} P_{n}^{(\beta, \alpha)}(\cos (\theta)) n^{-\alpha}\right) \\
& =\left\{\begin{array}{ll}
O\left(\frac{1}{n^{2+\alpha-\beta}}\right), & 0 \leq \theta \leq c n^{-1} \\
O\left(\theta^{-\beta+\frac{3}{2}} n^{-\frac{1}{2}} n^{-\alpha}\right)=O\left(n^{-\min \left\{2+\alpha-\beta, \alpha+\frac{1}{2}\right\}}\right), & c n^{-1} \leq \theta \leq \frac{\pi}{2}
\end{array} .\right.
\end{aligned}
$$

These together yield

$$
\left\|\ell_{0}\right\|_{\infty}=O\left(\frac{1}{n^{\min \left\{0,2+\alpha-\beta, \alpha+\frac{1}{2}\right\}}}\right)
$$

- Similarly, we have

$$
\left\|\ell_{n+1}\right\|_{\infty}=O\left(\frac{1}{n^{\min \left\{0,2+\beta-\alpha, \beta+\frac{1}{2}\right\}}}\right) .
$$

- For $k=1,2, \ldots, n$, let $x_{m}$ be the nearest to $t \in[0,1]$ and $t=\cos (\theta)$. From $(4.24)$, we have for $k \neq m$ that

$$
\ell_{k}(t)=\frac{\sin ^{2} \theta P_{n}^{(\alpha, \beta)}(\cos \theta)}{\left(\cos \theta-\cos \theta_{k}\right) \sin ^{2} \theta_{k} P_{n}^{(\alpha, \beta)^{\prime}}\left(\cos \theta_{k}\right)}=\frac{-\sin ^{2} \theta P_{n}^{(\alpha, \beta)}(\cos \theta)}{2 \sin \left(\frac{\theta-\theta_{k}}{2}\right) \sin \left(\frac{\theta+\theta_{k}}{2}\right) \sin ^{2} \theta_{k} P_{n}^{(\alpha, \beta)^{\prime}}\left(\cos \theta_{k}\right)} .
$$

In the case $0 \leq \theta \leq c n^{-1}$ and $0 \leq \theta_{k} \leq \frac{2 \pi}{3}$ : From (4.15)-(4.18), it follows

$$
(4.28) \ell_{k}(\cos \theta)=O\left(\frac{n^{-2} n^{\alpha}}{|k-m||k+m| n^{-2} k^{2} n^{-2} k^{-\alpha-\frac{3}{2}} n^{\alpha+2}}\right)=O\left(\frac{k^{\alpha-\frac{1}{2}}}{|k-m||k+m|}\right) .
$$

Define

$$
h_{1}(u)=\frac{u^{\alpha-\frac{1}{2}}}{u^{2}-m^{2}} \quad \text { for } m+1 \leq u \leq n ; \quad h_{2}(u)=-\frac{u^{\alpha-\frac{1}{2}}}{u^{2}-m^{2}} \quad \text { for } 1 \leq u \leq m-1 .
$$

Then by an elementary proof and noting that $m \leq c_{1} n$ for $0<c_{1}<1$, we get

$$
\max _{m+1 \leq u \leq n} h_{1}(u)= \begin{cases}h_{1}(m+1)=O\left(m^{\alpha-\frac{3}{2}}\right), & -1<\alpha \leq \frac{5}{2} \\ \max \left\{h_{1}(m+1), h_{1}(n)\right\}=O\left(\max \left\{m^{\alpha-\frac{3}{2}}, n^{\alpha-\frac{5}{2}}\right\}\right), & \alpha>\frac{5}{2}\end{cases}
$$


and

$\max _{1 \leq u \leq m-1} h_{2}(u)=\left\{\begin{array}{ll}\max \left\{h_{2}(1), h_{2}(m-1)\right\}=O\left(\max \left\{m^{-2}, m^{\alpha-\frac{5}{2}}\right\}\right), & -1<\alpha \leq \frac{1}{2} \\ h_{2}(m-1)=O\left(m^{\alpha-\frac{3}{2}}\right), & \alpha>\frac{1}{2}\end{array}\right.$,

which, together with $m \sim 1$ under the assumption, establishes that

$$
\ell_{k}(\cos \theta)=\left\{\begin{array}{ll}
O(1), & -1<\alpha \leq \frac{5}{2} \\
O\left(n^{\alpha-\frac{5}{2}}\right), & \alpha>\frac{5}{2}
\end{array} .\right.
$$

In the case $0 \leq \theta \leq c n^{-1}$ and $\frac{2 \pi}{3}<\theta_{k}<\pi$ : Similarly, from (4.15) and (4.20) we have

$$
\begin{aligned}
\ell_{k}(\cos \theta) & =O\left(\frac{n^{-2} n^{\alpha}}{(n-k+1)^{2} n^{-2}(n-k+1)^{-\beta-\frac{3}{2}} n^{\beta+2}}\right) \\
& =O\left(\frac{(n-k+1)^{\beta-\frac{1}{2}}}{n^{2+\beta-\alpha}}\right) \\
& =O\left(n^{-\min \left\{2+\beta-\alpha, \frac{5}{2}-\alpha\right\}}\right) .
\end{aligned}
$$

In the case $c n^{-1} \leq \theta \leq \frac{\pi}{2}$ and $0 \leq \theta_{k} \leq \frac{2 \pi}{3}$ : By (4.27), together with (4.15)-(4.18), we obtain

$$
\ell_{k}(\cos \theta)=O\left(\frac{m^{\frac{3}{2}-\alpha} k^{\alpha-\frac{1}{2}}}{|k-m||k+m|}\right)=m^{\frac{3}{2}-\alpha} O\left(\frac{k^{\alpha-\frac{1}{2}}}{|k-m||k+m|}\right)
$$

which establishes that by applying the estimates to $h_{1}(u)$ and $h_{2}(u)$

$$
\ell_{k}(\cos \theta)=\left\{\begin{array}{ll}
O\left(n^{-\alpha-\frac{1}{2}}\right), & -1<\alpha<-\frac{1}{2} \\
O(1), & -\frac{1}{2} \leq \alpha \leq \frac{5}{2} \\
O\left(n^{\alpha-\frac{5}{2}}\right), & \alpha>\frac{5}{2}
\end{array} .\right.
$$

In the case $c n^{-1} \leq \theta \leq \frac{\pi}{2}$ and $\frac{2 \pi}{3}<\theta_{k}<\pi$ : From (4.15)-(4.18), (4.20) and (4.27), we find that

$$
\ell_{k}(\cos \theta)=m^{\frac{3}{2}-\alpha} O\left(\frac{(n-k+1)^{\beta-\frac{1}{2}}}{n^{2+\beta-\alpha}}\right)=\left\{\begin{array}{ll}
O\left(n^{-\min \left\{0, \beta+\frac{1}{2}\right\}}\right), & -1<\alpha \leq \frac{3}{2} \\
O\left(n^{-\min \left\{2+\beta-\alpha, \frac{5}{2}-\alpha\right\}}\right), & \alpha>\frac{3}{2}
\end{array} .\right.
$$

Thus for $t \in[0,1]$, we get

$$
\left\|\ell_{k}\right\|_{\infty}=\left\{\begin{array}{ll}
O\left(n^{-\min \left\{0, \beta+\frac{1}{2}, \alpha+\frac{1}{2}\right\}}\right), & -1<\alpha \leq \frac{3}{2} \\
O\left(n^{-\min \left\{0,2+\beta-\alpha, \frac{5}{2}-\alpha\right\}}\right), & \alpha>\frac{3}{2}
\end{array} .\right.
$$

For $t \in[-1,0]$, by $P_{n}^{(\beta, \alpha)}(-t)=(-1)^{n} P_{n}^{(\alpha, \beta)}(t)$, setting $t=-\cos \theta$ and $y_{k}=-x_{n-k+1}=$ $\cos \bar{\theta}_{k}$ for $k=1,2, \ldots, n$, we see that $y_{k}$ are the roots of $P_{n}^{(\alpha, \beta)}(-t)=(-1)^{n} P_{n}^{(\beta, \alpha)}(t)$, then (4.24) can be represented as for $k=1,2, \ldots, n$

$$
\ell_{n-k+1}(t)=\frac{\left(1-\cos ^{2} \theta\right)(-1)^{n} P_{n}^{(\beta, \alpha)}(\cos \theta)}{-\left(\cos \theta-y_{k}\right)\left(1-y_{k}^{2}\right) P_{n}^{(\alpha, \beta)^{\prime}}\left(-y_{k}\right)}=\frac{\sin ^{2} \theta P_{n}^{(\beta, \alpha)}(\cos \theta)}{\left(\cos \theta-y_{k}\right)\left(1-y_{k}^{2}\right) P_{n}^{(\beta, \alpha)^{\prime}}\left(y_{k}\right)}
$$

followed

$$
\left[P_{n}^{(\alpha, \beta)}(t)\right]^{\prime}=\frac{1}{2}(n+\alpha+\beta+1) P_{n-1}^{(\alpha+1, \beta+1)}(t)[55,(4.21 .7)] .
$$


Similarly, we get that

$$
\left\|\ell_{k}\right\|_{\infty}=\left\{\begin{array}{ll}
O\left(n^{-\min \left\{0, \alpha+\frac{1}{2}, \beta+\frac{1}{2}\right\}}\right), & -1<\beta \leq \frac{3}{2} \\
O\left(n^{-\min \left\{0,2+\alpha-\beta, \frac{5}{2}-\beta\right\}}\right), & \beta>\frac{3}{2}
\end{array},\right.
$$

which together with (4.34) leads to that for $t \in[-1,1]$

$$
\left\|\ell_{k}\right\|_{\infty}=\left\{\begin{array}{ll}
O\left(n^{-\min \left\{0, \alpha+\frac{1}{2}, \beta+\frac{1}{2}\right\}}\right), & -1<\alpha, \beta \leq \frac{3}{2} \\
O\left(n^{-\min \left\{0, \alpha+\frac{1}{2}, 2+\alpha-\beta, \frac{5}{2}-\beta\right\}}\right), & -1<\alpha \leq \frac{3}{2}, \beta>\frac{3}{2} \\
O\left(n^{-\min \left\{0, \beta+\frac{1}{2}, 2+\beta-\alpha, \frac{5}{2}-\alpha\right\}}\right), & \alpha>\frac{3}{2},-1<\beta \leq \frac{3}{2} \\
O\left(n^{-\min \left\{0,2+\alpha-\beta, 2+\beta-\alpha, \frac{5}{2}-\alpha, \frac{5}{2}-\beta\right\}}\right), & \alpha, \beta>\frac{3}{2}
\end{array} .\right.
$$

THEOREM 4.5. Suppose $f(t)$ satisfies (2.9) and $\left\{x_{j}\right\}_{j=0}^{n+1}$ are the roots of $\left(1-t^{2}\right) P_{n}^{(\alpha, \beta)}(t)$, then for $n \geq r+1$

$$
E_{n}[f]=n^{-r} \cdot\left\{\begin{array}{ll}
O\left(n^{-\min \left\{0, \alpha+\frac{1}{2}, \beta+\frac{1}{2}\right\}}\right), & -1<\alpha, \beta \leq \frac{3}{2} \\
O\left(n^{-\min \left\{0, \alpha+\frac{1}{2}, 2+\alpha-\beta, \frac{5}{2}-\beta\right\}}\right), & -1<\alpha \leq \frac{3}{2}, \beta>\frac{3}{2} \\
O\left(n^{-\min \left\{0, \beta+\frac{1}{2}, 2+\beta-\alpha, \frac{5}{2}-\alpha\right\}}\right), & \alpha>\frac{3}{2},-1<\beta \leq \frac{3}{2} \\
O\left(n^{-\min \left\{0,2+\alpha-\beta, 2+\beta-\alpha, \frac{5}{2}-\alpha, \frac{5}{2}-\beta\right\}}\right), & \alpha, \beta>\frac{3}{2}
\end{array} .\right.
$$

Particularly, we have for $(\alpha, \beta) \in S$

$$
\left\|E_{n}[f]\right\|_{\infty}=O\left(\frac{1}{n^{r}}\right)
$$

where $S:=\left[-\frac{1}{2}, \frac{5}{2}\right] \times\left[-\frac{1}{2}, \frac{5}{2}\right]-\left\{(\alpha, \beta):-\frac{1}{2} \leq \alpha \leq \frac{1}{2}, 2+\alpha<\beta \leq \frac{5}{2}\right\} \cup\left\{(\alpha, \beta): \frac{3}{2} \leq \alpha \leq\right.$ $\left.\frac{5}{2},-\frac{1}{2} \leq \beta<2-\alpha\right\}$.

REMARK 3. Theorem 4.5 implies $\left\|f-L_{n}[f]\right\|_{\infty}$ has the same asymptotic order as $\| f-$ $p_{n-1}^{*} \|_{\infty}$ [62] at the roots of the Jacobi polynomial $\left(1-t^{2}\right) P_{n}^{(\alpha, \beta)}(t)$ for $(\alpha, \beta) \in S$, which includes the corresponding strongly normal pointsystems as special cases.

Figs. 4.9-4.10 show the convergence rates for $f(x)=|x|$ or $f(x)=|x|^{3}$ at the Jacobi-GaussLobatto pointsystems, respectively, where each $(\alpha, \beta)$ is generated by $2 \operatorname{rand}(1,2)-0.5$.

\subsection{General Jacobi-Gauss-Radau pointsystems. Let}

$$
-1<x_{n}<x_{n-1}<\cdots<x_{2}<x_{1}<x_{0}=1
$$

be the roots of $(1-t) P_{n}^{(\alpha, \beta)}(t)=0(\alpha, \beta>-1), x_{k}=\cos \left(\theta_{k}\right)$ and

$$
\omega(t)=\left(t-x_{0}\right)\left(t-x_{1}\right) \cdots\left(t-x_{n}\right), \quad \ell_{k}(t)=\frac{\omega(t)}{\left(t-x_{k}\right) \omega^{\prime}\left(x_{k}\right)} .
$$

Then

$$
\ell_{0}(t)=\frac{P_{n}^{(\alpha, \beta)}(t)}{P_{n}^{(\alpha, \beta)}(1)}, \quad \ell_{k}(t)=\frac{(1-t) P_{n}^{(\alpha, \beta)}(t)}{\left(t-x_{k}\right)\left(1-x_{k}\right) P_{n}^{(\alpha, \beta)^{\prime}}\left(x_{k}\right)}, \quad k=1,2, \ldots, n .
$$

Additionally, for $t=\cos \theta \in[0,1]$, we have

$$
\ell_{0}(t)=\left\{\begin{array}{ll}
O\left(\frac{n^{\alpha}}{n^{\alpha}}\right), & 0 \leq \theta \leq c n^{-1} \\
O\left(\theta^{-\alpha-\frac{1}{2}} n^{-\alpha-\frac{1}{2}}\right), & c n^{-1} \leq \theta<\frac{\pi}{2}
\end{array}=O\left(n^{-\min \left\{0, \alpha+\frac{1}{2}\right\}}\right),\right.
$$



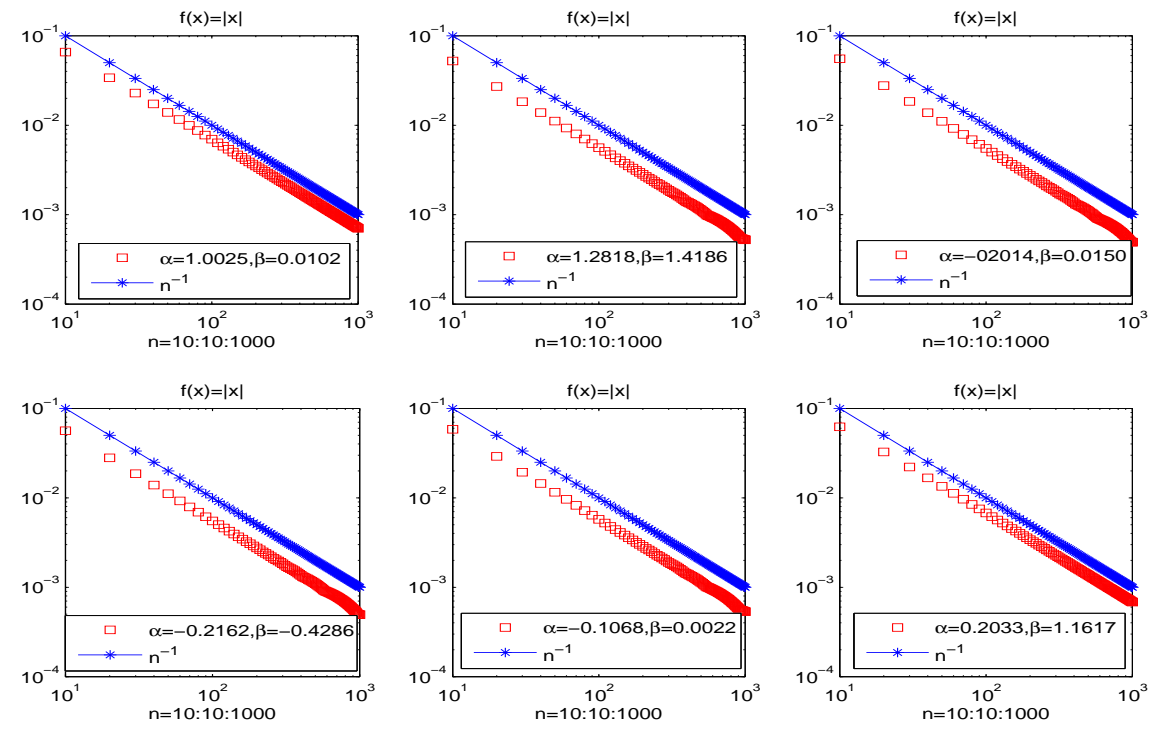

FIG. 4.9. $\max _{x=-1: 0.001: 1}\left|f(x)-L_{n}[f](x)\right|$ with $n=10: 10: 1000$ at the Jacobi-Gauss-Lobatto pointsystems for $f(x)=|x|$, respectively.
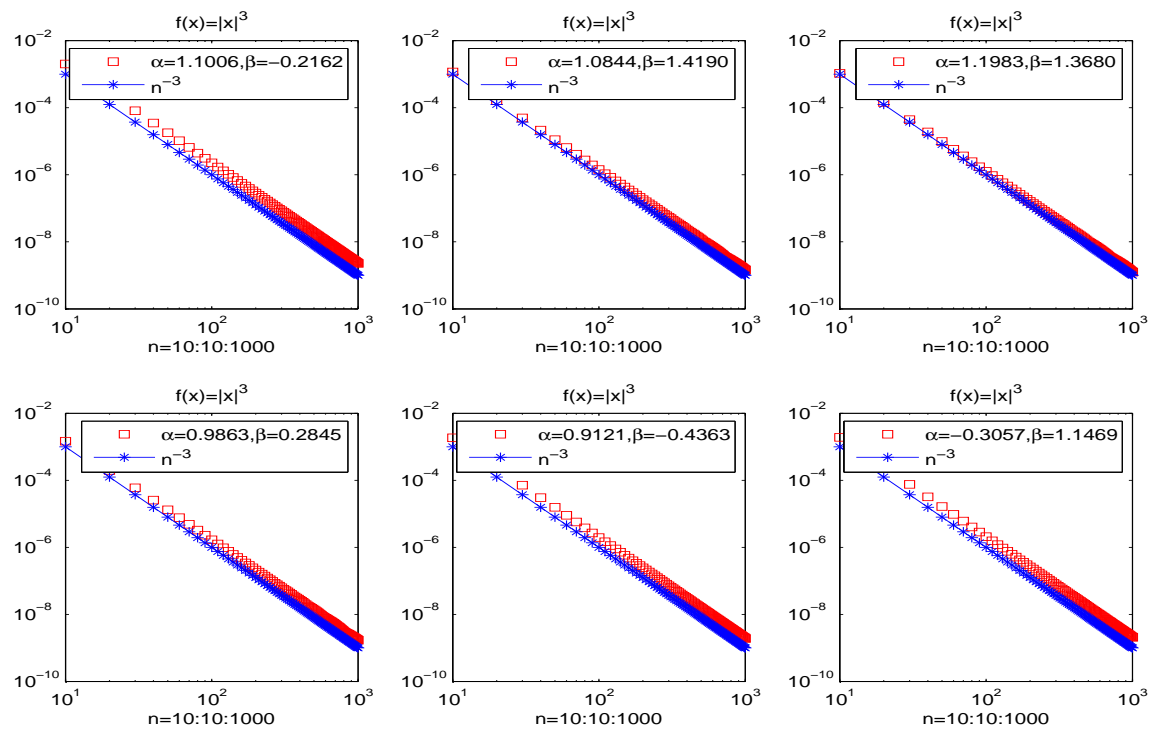

FIG. 4.10. $\max _{x=-1: 0.001: 1}\left|f(x)-L_{n}[f](x)\right|$ with $n=10: 10: 1000$ at the Jacobi-Gauss-Lobatto pointsystems for $f(x)=|x|^{3}$, respectively.

and

$$
\begin{aligned}
\ell_{k}(t) & =-\frac{2 \sin ^{2}(\theta / 2) P_{n}^{(\alpha, \beta)}(\cos \theta)}{2 \sin ^{2}\left(\theta_{k} / 2\right) P_{n}^{(\alpha, \beta)^{\prime}}\left(\cos \theta_{k}\right) 2 \sin \left(\left(\theta-\theta_{k}\right) / 2\right) \sin \left(\left(\theta+\theta_{k}\right) / 2\right)} \\
& = \begin{cases}O\left(n^{-\min \left\{0, \alpha+\frac{1}{2}\right\}}\right), & -1<\alpha \leq-\frac{1}{2} \\
O\left(n^{-\min \left\{0, \frac{5}{2}-\alpha\right\}}\right), & \alpha>-\frac{1}{2}\end{cases}
\end{aligned}
$$

following (4.27) similarly. 
Similarly, for $t \in[-1,0]$, by $P_{n}^{(\beta, \alpha)}(-t)=(-1)^{n} P_{n}^{(\alpha, \beta)}(t)$, setting $t=-\cos \theta$ and $y_{k}=$ $-x_{n-k+1}=\cos \bar{\theta}_{k}$ for $k=1,2, \ldots, n$, we obtain $\ell_{0}(t)=O\left(n^{-\min \left\{\alpha+\frac{1}{2}, \alpha-\beta\right\}}\right)$ and

$$
\ell_{k}(t)=\left\{\begin{array}{ll}
O\left(n^{-\min \left\{0, \alpha+\frac{1}{2}, \alpha-\beta\right\}}\right), & -1<\alpha<\frac{1}{2} \\
O\left(n^{-\min \left\{0, \frac{1}{2}-\beta\right\}}\right), & \alpha \geq \frac{1}{2}
\end{array} .\right.
$$

Thus for $t \in[-1,1]$, we get

$$
\left\|\ell_{k}\right\|_{\infty}= \begin{cases}O\left(n^{-\min \left\{0, \alpha+\frac{1}{2}, \alpha-\beta\right\}}\right), & -1<\alpha \leq \frac{1}{2} \\ O\left(n^{-\min \left\{0, \frac{1}{2}-\beta, \frac{5}{2}-\alpha, \alpha-\beta\right\}}\right), & \alpha>\frac{1}{2}\end{cases}
$$

for $k=0,1,2, \ldots, n$.

THEOREM 4.6. Suppose $f(t)$ satisfies (2.9) and $\left\{x_{j}\right\}_{j=0}^{n}$ are the roots of $(1-t) P_{n}^{(\alpha, \beta)}(t)$, then for $n \geq r+1$

$$
E_{n}[f]=n^{-r} \cdot \begin{cases}O\left(n^{-\min \left\{0, \alpha+\frac{1}{2}, \alpha-\beta\right\}}\right), & -1<\alpha \leq \frac{1}{2} \\ O\left(n^{-\min \left\{0, \frac{1}{2}-\beta, \frac{5}{2}-\alpha, \alpha-\beta\right\}}\right), & \alpha>\frac{1}{2}\end{cases}
$$

Particularly, we have for $(\alpha, \beta) \in \bar{S}$

$$
\left\|E_{n}[f]\right\|_{\infty}=O\left(\frac{1}{n^{r}}\right)
$$

where $\bar{S}:=\left[-\frac{1}{2}, \frac{5}{2}\right] \times\left(-1, \frac{1}{2}\right]-\left\{(\alpha, \beta):-\frac{1}{2} \leq \alpha \leq \frac{1}{2}, \alpha<\beta \leq \frac{1}{2}\right\}$.

Similarly we have

THEOREM 4.7. Suppose $f(t)$ satisfies (2.9) and $\left\{x_{j}\right\}_{j=0}^{n}$ are the roots of $(1+t) P_{n}^{(\alpha, \beta)}(t)$, then for $n \geq r+1$

$$
E_{n}[f]=n^{-r} \cdot \begin{cases}O\left(n^{-\min \left\{0, \beta+\frac{1}{2}, \beta-\alpha\right\}}\right), & -1<\beta \leq \frac{1}{2} \\ O\left(n^{-\min \left\{0, \frac{1}{2}-\alpha, \frac{5}{2}-\beta, \beta-\alpha\right\}}\right), & \beta>\frac{1}{2}\end{cases}
$$

Particularly, we have for $(\alpha, \beta) \in \widehat{S}$

$$
\left\|E_{n}[f]\right\|_{\infty}=O\left(\frac{1}{n^{r}}\right)
$$

where $\widehat{S}:=\left(-1, \frac{1}{2}\right] \times\left[-\frac{1}{2}, \frac{5}{2}\right]-\left\{(\alpha, \beta):-\frac{1}{2} \leq \beta \leq \frac{1}{2}, \beta<\alpha \leq \frac{1}{2}\right\}$.

FIG. 4.11 shows the convergence rates for $f(x)=|x|$ at the Jacobi-Gauss-Radau pointsystems, where each $(\alpha, \beta) \in \bar{S}$ or $(\alpha, \beta) \in \widehat{S}$.

5. Final remarks. The results in section 4 indicate the fact that the interpolations, for functions of limited regularities, at strongly normal pointsystems, Gauss-Jacobi pointsystems with $-1<\alpha, \beta \leq \frac{1}{2}$, Jacobi-Gauss-Lobatto pointsystems with $(\alpha, \beta) \in S$, Gauss-Jacob-Radau pointsystems $\bar{S}$ or $\widehat{S}$, have the same convergence order compared with the best polynomial approximation of the same degree. Numerical experiments give in line with the estimates.

In addition, numerical experiments also show that the same occurs for analytic or smooth functions. Here we illustrate the phenomenons by entire function $f(x)=e^{x}$, i.e., analytic throughout the complex plane, $f(x)=1 /\left(1+25 x^{2}\right)$, which is analytic in a neighborhood of $[-1,1]$ but not throughout the complex plane, and $f(x)=e^{-1 / x^{2}}$, which is not analytic in a neighborhood of $[-1,1]$ but is infinitely differentiable in $[-1,1]$.

In Figs. 5.1-5.3, the left columns are computed by zeros of Gauss-Jacobi polynomial $P_{n}^{(\alpha, \beta)}(x)$, the middles by Jacobi-Gauss-Lobatto $\left(1-x^{2}\right) P_{n-2}^{(\alpha, \beta)}(x)$, while the rights by JacobiGauss-Radau $(1-x) P_{n-1}^{(\alpha, \beta)}(x)$ (first three cases) or $(1+x) P_{n-1}^{(\alpha, \beta)}(x)$ (last three cases), respectively. From these figures, we see that the interpolations at these pointsystems including the 

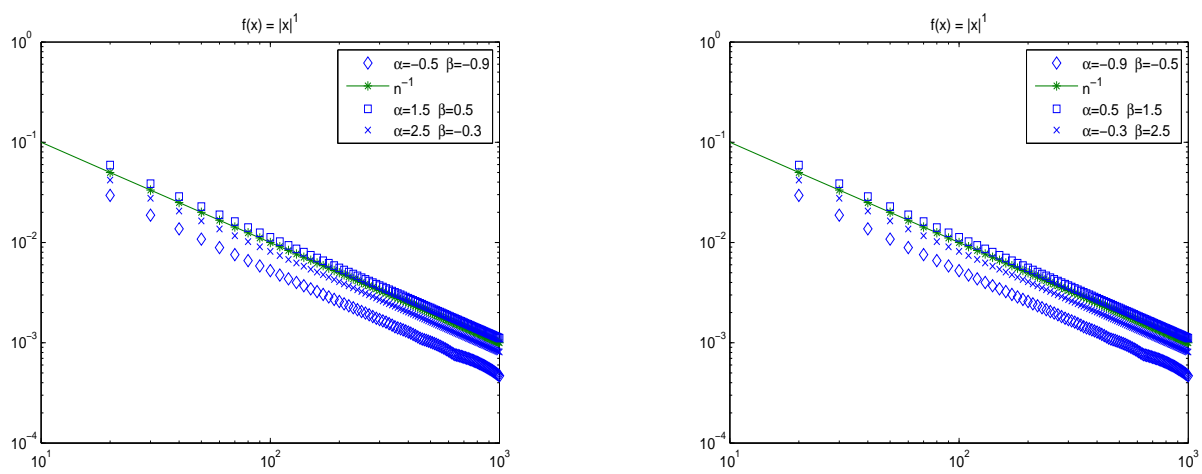

FIG. 4.11. The absolute errors of $\max _{x=-1: 0.001: 1}\left|f(x)-L_{n}[f](x)\right|$ for Jacobi-Gauss-Radau pointsystems: the roots of $(1-t) P_{n}^{(\alpha, \beta)}(t)$ (left) and the roots of $(1+t) P_{n}^{(\alpha, \beta)}(t)$ (right) for $f(x)=|x|$, respectively.
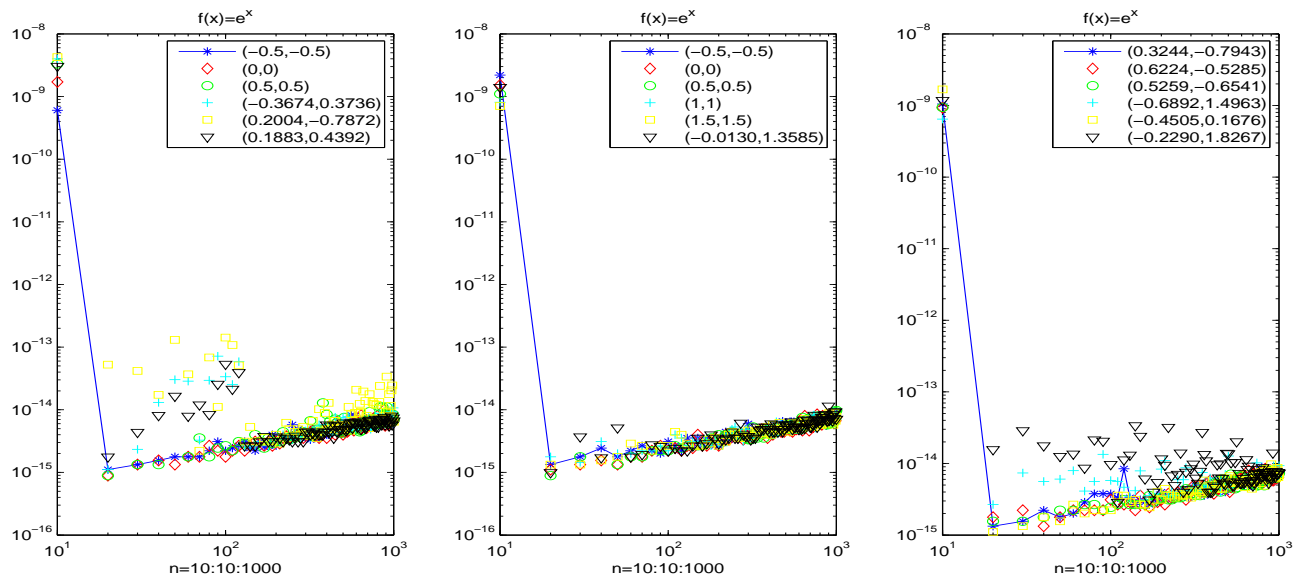

FIG. 5.1. $\max _{x=-1: 0.001: 1}\left|f(x)-L_{n}[f](x)\right|$ with $n=10: 10: 1000$ at Gauss-Jacobi pointsystems for $f(x)=e^{x}$, respectively.
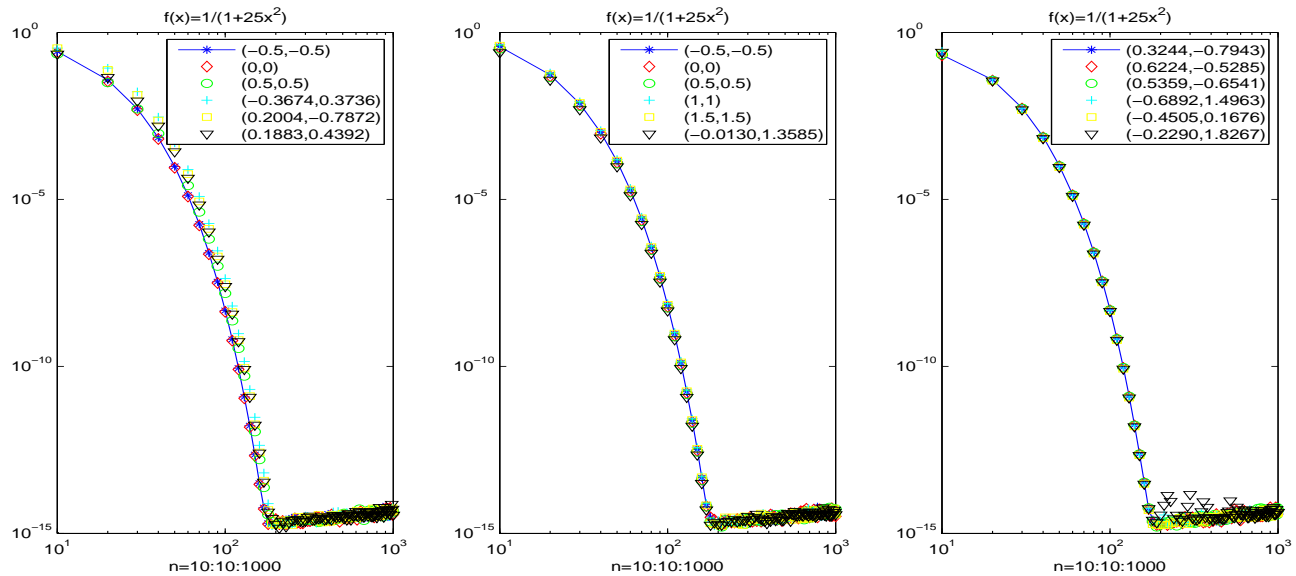

Fig. 5.2. $\max _{x=-1: 0.001: 1}\left|f(x)-L_{n}[f](x)\right|$ with $n=10: 10: 1000$ at Gauss-Jacobi pointsystems for $f(x)=\frac{1}{1+25 x^{2}}$, respectively . 

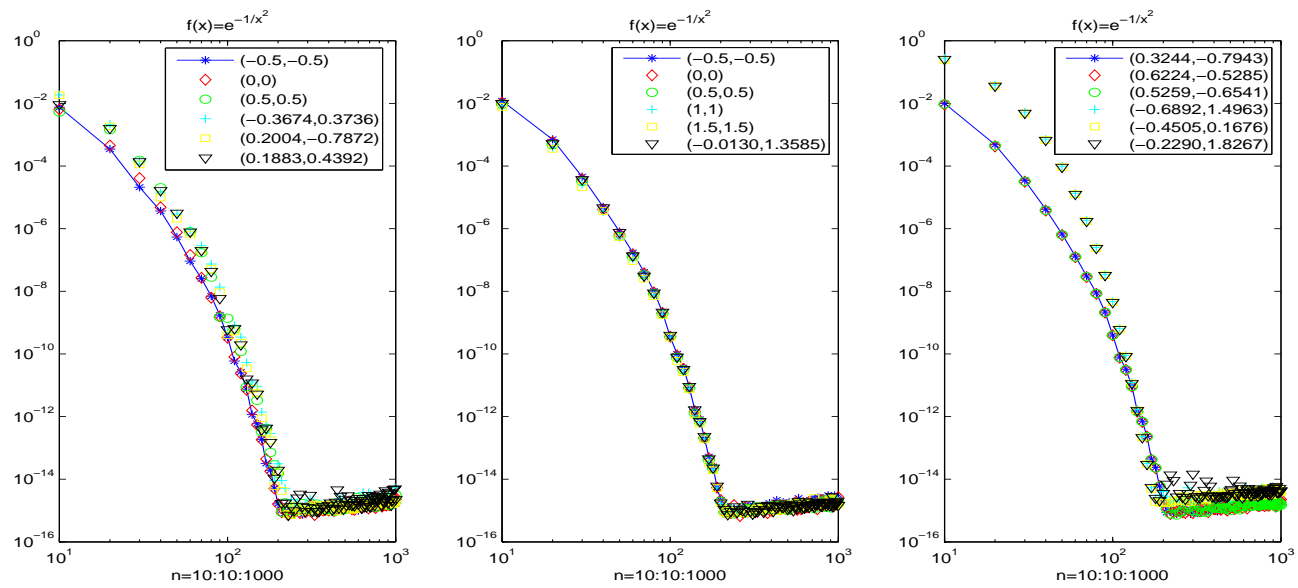

FIG. 5.3. $\max _{x=-1: 0.001: 1}\left|f(x)-L_{n}[f](x)\right|$ with $n=10: 10: 1000$ at Jacobi-Gauss-Radau pointsystems for $f(x)=e^{-1 / x^{2}}$, respectively.

Gauss-Legendre and Legendre-Gauss-Lobatto, achieve essentially the same approximation accuracy compared with those at the two Chebyshev piontsystems too.

It is interesting to noting that the interpolation approximation polynomial $L_{n}[f]$ challenges the best approximation polynomial $p_{n-1}^{*}$ of $f$ at the above nice pointsystems not only on the equally asymptotic order on the convergence rate, but also on the faster convergence on the first derivative or second derivative approximation by the polynomials, which has plenty applications in spectral methods 31, 57.

FIG. 5.4 illustrates that the convergence rates $\max _{x=-1: 0.001: 1}\left|f^{\prime}(x)-\left[p_{n-1}^{*}\right]^{\prime}(x)\right|$ and $\max _{x=-1: 0.001: 1}\left|f^{\prime \prime}(x)-\left[p_{n-1}^{*}\right]^{\prime \prime}(x)\right|$ reduces 2-order and 3-order, respectively, compared with $\max _{x=-1: 0.001: 1}\left|f(x)-p_{n-1}^{*}(x)\right|$ for $f(x)=|x|^{5}$ or $|x|^{7}$. Here, the best approximation polynomial $p_{n-1}^{*}$ is obtained by remez algorithm in CheBfun system [60].

However, the interpolation polynomial $L_{n}[f]$ at the above nice pointsystems performs much better than $p_{n-1}^{*}$ for approximation $f^{\prime}$ and $f^{\prime \prime}$ by $L_{n}^{\prime}[f], L_{n}^{\prime \prime}[f],\left[p_{n-1}^{*}\right]^{\prime}$ and $\left[p_{n-1}^{*}\right]^{\prime \prime}$, respectively. Here, we use usual pointsystems to show the performs (see Figs. 5.5-5.7).
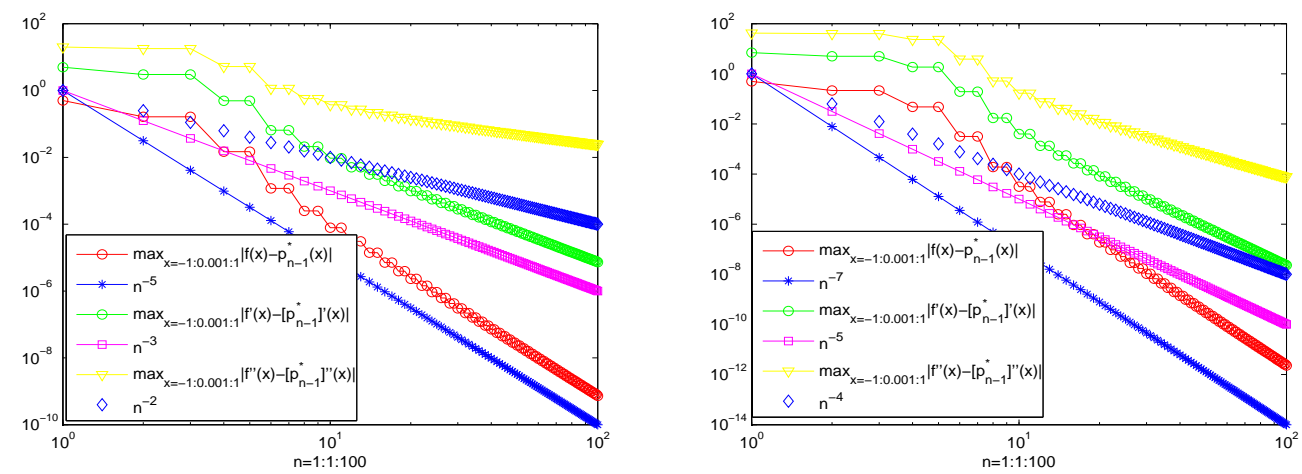

FIG. 5.4. $\max _{x=-1: 0.001: 1}\left|f^{(m)}(x)-\left[p_{n-1}^{*}\right]^{(m)}(x)\right|$ with $n=10: 1: 100$ for $f(x)=|x|^{5}$ (left), $f(x)=|x|^{7}$ (right) and $m=0,1,2$, respectively.

Fig. 5.8 shows that the convergence rates $\max _{x=-1: 0.001: 1}\left|f^{(m)}(x)-\left[p_{n-1}^{*}\right]^{(m)}(x)\right|$ and $\max _{x=-1: 0.001: 1}\left|f^{(m)}(x)-L_{n}^{(m)}[f](x)\right|$ for $f(x)=|x|^{5}$ and $|x|^{7}$, respectively, where $L_{n}[f]$ is the interpolation at Jacobi-Gauss-Lobatto points $\left\{x_{k}=\cos \left(\frac{k \pi}{n-1}\right)\right\}_{k=0}^{n-1}$.

Acknowledgement. The author is grateful to Professor Kelzon for his kind help, and to 

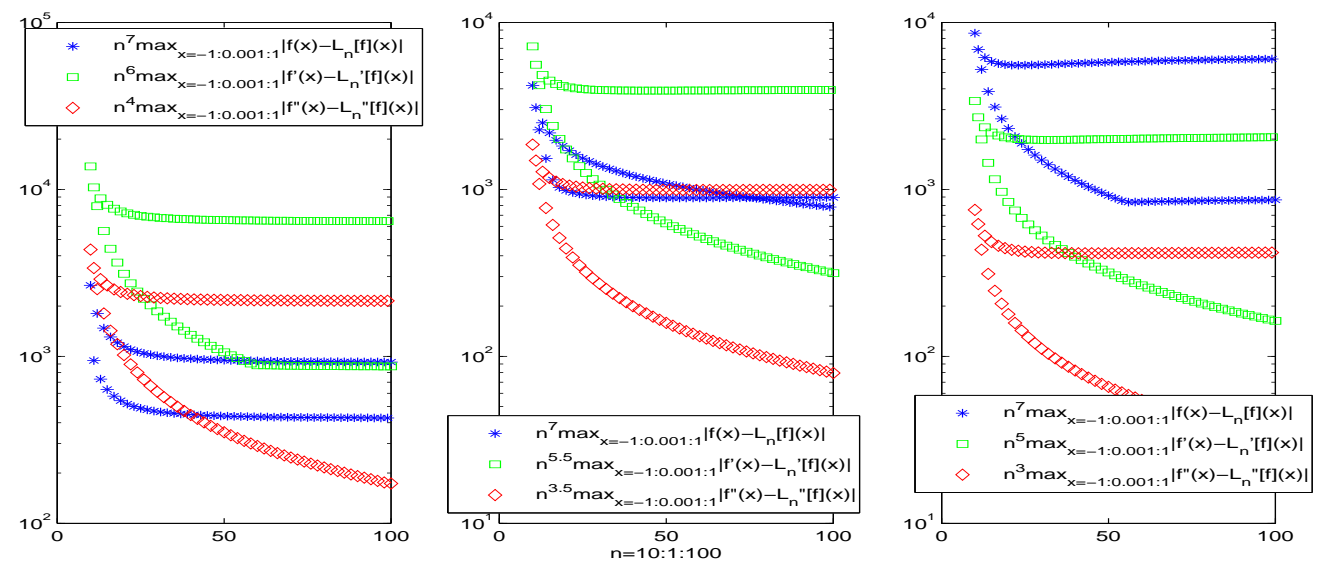

FIG. 5.5. $n^{s(m)} \max _{x=-1: 0.001: 1}\left|f^{(m)}(x)-L_{n}^{(m)}[f](x)\right|$ with $n=10: 1: 100$ at Gauss-Jacobi pointsystems for $f(x)=|x|^{7}$ and $m=0,1,2$, and $(-0.5,-0.5)$ (left), $(0,0)$ (middle), (0.5,0.5) (right), respectively.
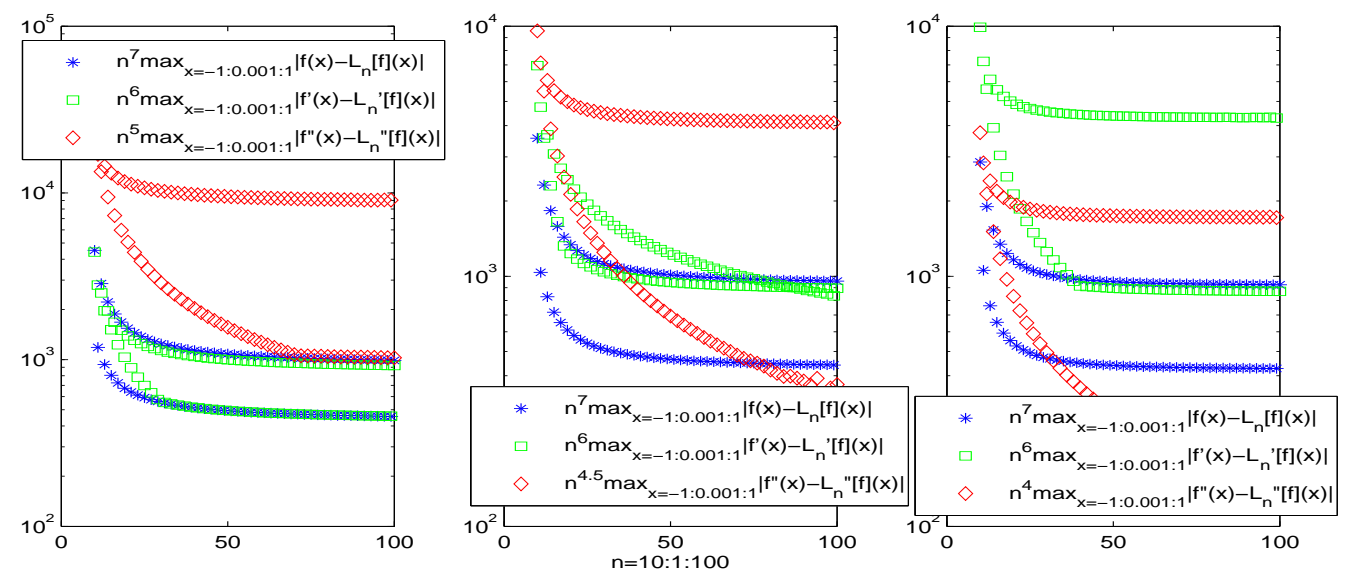

FIG. 5.6. $n^{s(m)} \max _{x=-1: 0.001: 1}\left|f^{(m)}(x)-L_{n}^{(m)}[f](x)\right|$ with $n=10: 1: 100$ at Jacobi-Gauss-Lobatto pointsystems for $f(x)=|x|^{7}$ and $m=0,1,2$, and (0.5,0.5) (left), (1,1) (middle), (1.5,1.5) (right), respectively.
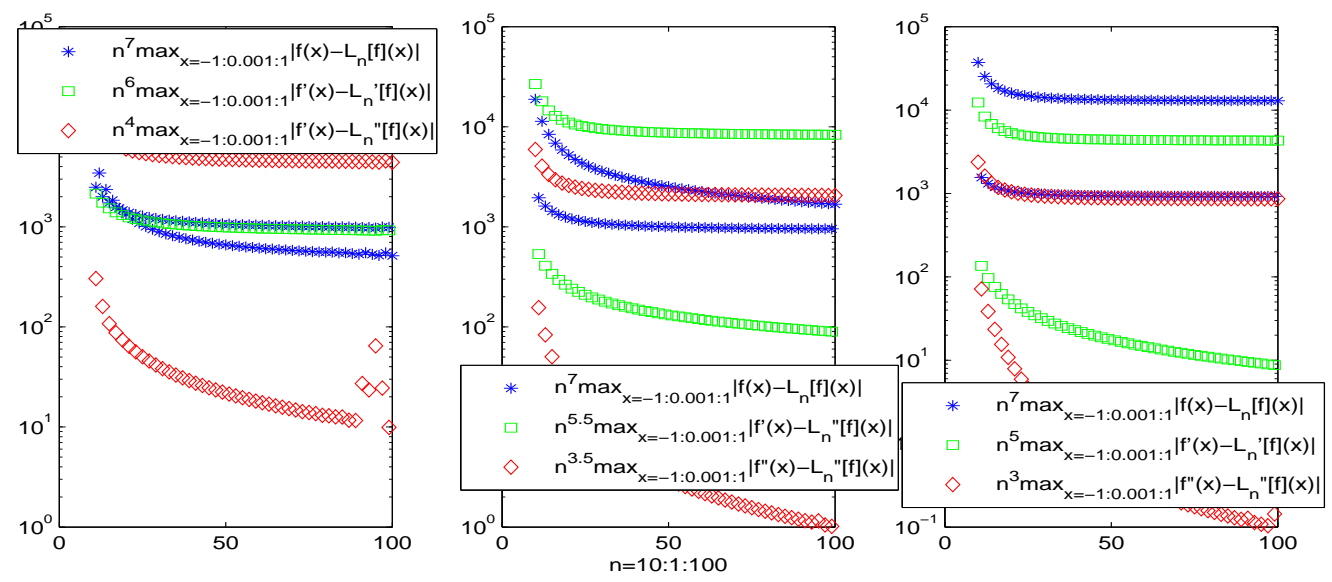

FIG. 5.7. $n^{s(m)} \max _{x=-1: 0.001: 1}\left|f^{(m)}(x)-L_{n}^{(m)}[f](x)\right|$ with $n=10: 1: 100$ at Jacobi-Gauss-Radau pointsystems with $x_{0}=1$ for $f(x)=|x|^{7}$ and $m=0,1,2$, and $(-0.5,-0.5)$ (left), (0,0) (middle), (0.5,0.5) (right), respectively. 

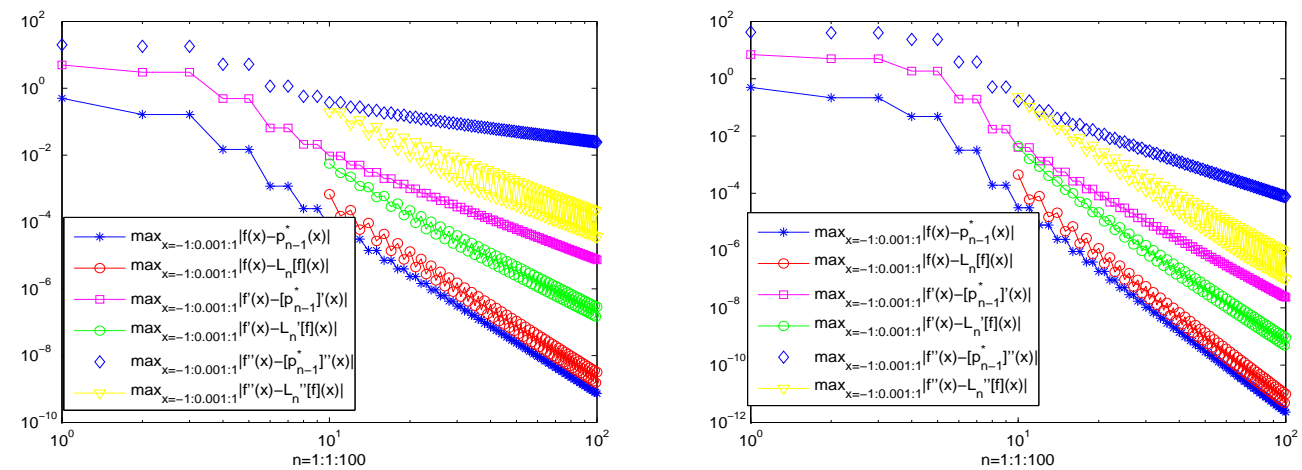

FIG. 5.8. The convergence rates $\max _{x=-1: 0.001: 1}\left|f^{(m)}(x)-\left[p_{n-1}^{*}\right]^{(m)}(x)\right|$ and $\max _{x=-1: 0.001: 1} \mid f^{(m)}(x)-$ $L_{n}^{(m)}[f](x) \mid$ for $f(x)=|x|^{5}$ (left) and $|x|^{7}$ (right) with $m=0,1,2$, respectively, where $L_{n}[f]$ is the interpolation at Jacobi-Gauss-Lobatto points $\left\{x_{k}=\cos \left(\frac{k \pi}{n-1}\right)\right\}_{k=0}^{n-1}$.

Professor Yuri Wainerman for her sending me three pages of her $\mathrm{Ph}$. D thesis including the interesting Lemma 3.1. The author thanks Chaoxu Pei at Florida State University and Yulong $\mathrm{Lu}$ at University of Warwick for their checking up every detail and constructive comments. The author also thanks Dr. Guo He and Guidong Liu, at Central South University, for their helpful and insightful discussion on the convergence rates of the derivatives of interpolations and estimats on the Jacobi-Gauss-Radau pointsystems.

\section{REFERENCES}

[1] S. Bernstein, Sur l'ordre de la meilleure approximation des fonctions continues par les polynômes de degré donné, Mem. Cl. Sci. Acad. Roy. Belg., 4 (1912), 1-103.

[2] S. N. Bernstein, Quelques remarques sur l'interpolation, Comm. Soc. Math. Charkow, 14 (1914).

[3] S. N. Bernstein (1914B), Sur la meilleure approximation de $|x|$ par des polynômes de degrés donnés, Acta Math. 37 (1914), 1-57.

[4] J. P. Berrut and L.N. Trefethen, Barycentric Lagrange interpolation, SiAM Rev., 46 (2004), 501-517.

[5] I. Bogaert, B. Michiels and J. Fostier, O(1) Computation of Legendre Polynomials and Gauss-Legendre Nodes and Weights for Parallel Computing, SIAM J. Sci. Comput., 34 (2012), C83-C101.

[6] H. Brass and K. Petras, Quadrature Theory, Amer. Math. Soc., Providence, RI, 2011.

[7] L. Brutman, On the Lebesgue function for polynomial interpolation, SIAM J. Numer. Anal., 15 (1978), 694-704.

[8] L. Brutman, Lebesgue functions for polynomial interpolation-a survey, Ann. Numer. Math., 4(1997), 111127.

[9] E. W. Cheney, Introduction to Approximation Theory, McGraw-Hill, New York, 1966.

[10] C. W. Clenshaw and A. R. Curtis, A method for numerical integration on an automatic computer, Numer. Math., 2(1960) 197-205.

[11] G. DAhlquist And Å. BJörck, Numerical Methods in Scientific Computing, SIAM, Philadelphia, 2008.

[12] P. J. DAVIS, Interpolation and Approximation, Dover, 1975.

[13] Z. Ditzian And V. Totik, Moduli of Smoothness, Springer, New York, 1987.

[14] H. Ehlich And K. Zeller, Auswertung der Normen von Interpolationsoperatoren, Math. Ann., 164 (1966), 105-112.

[15] P. ERDös, Problems and results on the theory of interpolation. II, Acta Math. Acad. Sci. Hungar., 12 (1961), 235-244.

[16] P. ERdös ANd P. TuRÁn, On interpolation II: On the distribution of the fundmental points of Lagrange and Hermite interpolation, Ann. Math., 39 (1938), 703-724.

[17] P. ERdös And P. TuRÁN, On interpolation. III. Interpolation theory of polynomials, Ann. Math., 41 (1940), 510-533.

[18] G. FABER, Über die interpolatorische Darstellung stetiger Funktionen, Jahresber. Deut. Math. Verein. 23 (1914), 192-210.

[19] L. FEJÉR, Über Interpolation, Nachrichten der Gesellschaft der Wissenschaften zu Göttingen Mathematischphysikalische Klasse, 1916, 66-91.

[20] L. FEJÉR, Lagrangesche interpolation und die zugehörigen konjugierten Punkte, Math. Ann., 106(1932), $1-55$.

[21] L. FEJÉR, Bestimmung derjenigen Abszissen eines Intervalles, für welche die Quadratsumme der Grundfunktionen der Lagrangeschen Interpolation im Intervalle ein Möglichst kleines Maximum Besitzt, An- 
nali della Scuola Norm sup. di Pisa, 1 (1932), 263-276.

[22] L. Gatteschi, New inequalities for the zeros of Jacobi polynomials, SIAM J. Math. Anal., 18 (1987) 15491562.

[23] A. Glaser, X. Liu and V. Rokhlin, A fast algorithm for the calculation of the roots of special functions, SIAM J. Sci. Comput., 29 (2007), 1420-1438.

[24] G. GRÜNwALD, Über Divergenzerscheinungen tier Lagrangeschen Interpolationspolynome, Acta Szeged, 7 (1935), 207-211.

[25] G. Grünwald, On the theory of interpolation, Acta Math., 75(1942), 219-245.

[26] N. Hale And A. Townsend, Fast and accurate computation of Gauss-Legendre and Gauss- Jacobi quadrature nodes and weights, SIAM J. Sci. Comput., 35(2013), A652-A674.

[27] N. Hale and L. N. Trefethen, Chebfun and numerical quadrature, SIAM J. Numer. Anal., 46(2008), 930-948.

[28] N. Hale and L. N. Trefethen, Chebfun and numerical quadrature, Science in China, 55 (2012), 1749-1760.

[29] H. O'Hara and F. J. Smith, Error estimation in the Clenshaw-Curtis quadrature formula, Comp. J., 11(1968) 213-219.

[30] P. Henrici, Essentials of Numerical Analysis, Wiley, New York, 1982.

[31] J. Hesthaven, S. Gottlieb and D. Gottlieb, Spectral Methods for Time-Dependent Problems, Cambridge University Press, 2007.

[32] N. J. Higham, Accuracy and Stability of Numerical Algorithms, 2nd ed., SIAM, Philadelphian, 2002.

[33] N. J. Higham, The numerical stability of barycentric Lagrange interpolation, IMA J. Numer. Anal., 24 (2004), 547-556.

[34] A. A. Kelzon, Interpolation of functions of bounded p-variation, Izv. Vuzov. Matem., 5(1978), 131-134 (Russian).

[35] A. A. Kelzon, On interpolation of continuous functions of bounded p-variation, Izv. Vuzov. Matem., 8(1984), 14-20 (Russian).

[36] G. KowalEwski, Interpolation und genäherte Quadratur. Teubner-Verlag, Leipzig, 1932.

[37] V. I. KRYlov, Approximate Calculation of Integrals, Dover, 1962.

[38] G. Kvernadze, Uniform convergence of Lagrange interpolation based on the Jacobi nodes, J. Approx. Theory, 87(1996), 179-193.

[39] S. LANG, Real and Functional Analysis, 3rd Edition, Springer, 1997.

[40] F. Locher, On Hermite-Fejer interpolation at Jacobi zeros, J. Approx. Theory, 44(1985), 154-166.

[41] D. S. Lubinsky, A taste of Erdös on interpolation, in: Paul Erdös and his mathematics, I (Budapest, 1999), 423-454. Bolyai Soc. Math. Stud., 11, Janos Bolyai Math. Soc., Budapest, 2002.

[42] G. Mastroianni and J. Szabados, Jackson order of approximation by Lagrange interpolation. II, Acta Math. Acad. Sci. Hungar. 69 (1995), 73-82.

[43] J. Marcinkiewicz, Sur la divergence des polynomes d'interpolation, Acta Szeged, 8(1937), 131-135.

[44] V.R. MisEs, Über allgemeine Quadraturformeln, J. Reine Angew. Math., 174(1936), 56-67.

[45] G. Peano, Resto nelle formule di quadrature, espresso con un integrale definito, Rom. Acc. L. Rend., 22(1913), 562-569.

[46] J. Prestin, Lagrange interpolation for functions of bounded variation, Acta Math. Hung., 62(1993), 1-13.

[47] T. J. RivLin, The Lebesgue constants for polynomial interpolation, in Functional Analysis and its Applications, H.C. Garnier et al. eds., Springer-Verlag, Berlin, 1974, 422-437.

[48] W. Rudin, Real and Complex Analysis, McGraw-Hill Companies, Inc., 3rd Edition, 1987.

[49] C. Runge, Über empirische Funktionen und die Interpolation zwischen äquidistanten Ordinaten, Z. Math. Phys. 46 (1901), 224-243.

[50] H.E. SAlzer, Lagrangian interpolation at the Chebyshev points $x_{n, v}=\cos (v \pi / n), v=O(1) n$; some unnoted advantages, Comput. J., 15 (1972), 156-159.

[51] R. Schмidt, Die allgemeine Newtonsche Quadraturformel und Quadraturformeln fur Stieltjesintegrale, J. reine Angew. Math., 173(1935), 52-59.

[52] A. Schönhage, Fehlerfortpflanzung bei Interpolation, Numer. Math., 3 (1961), 62-71.

[53] E. Stein and R. Shakarchi, Real Analysis: Measure Theory, Integration, and Hilbert Spaces, Princeton University Press, 2005

[54] X. Sun, Lagrange interpolation of functions of generalized bounded variation, Acta Math. Hungar., 53(1989), 75-84.

[55] G. Szegö, Orthogonal Polynomials, Colloquium Publications 23, A, Providence, Rhode Island, 1939.

[56] T. TAO, An Introduction to Measure Theory, American Mathematical Society, 2011.

[57] L. N. Trefethen, Spectral Methods in MATLAB, SIAM, Philadelphia, 2000.

[58] L. N. Trefethen, Is Gauss quadrature better than Clenshaw-Curtis?, SIAM Rev., 50(2008), 67-87.

[59] L. N. Trefethen, Approximation Theory and Approximation in Practice, SIAM, Philadelphia, 2012.

[60] L. N. Trefethen And Others, Chebfun Version 4.0, The Chebfun Development Team, http://www.maths.ox.ac.uk/chebfun/ 2011.

[61] L. N. TREFEthen And J. A. C. Weideman, Two results concerning polynomial interpolation in equally spaced points, J. Approx. Th. 65 (1991), 247-260.

[62] Ch.-J. de la Vallée Poussin, Note sur l'approximation par un polynôme d'une fonction dont la derivée est à variation bornée, Bull. Acad. Belg. 1908, 403-410.

[63] R. S. Varga and A. J. Carpenter, On the Bernstein conjecture in approximation theory, Constr. Approx. 1 (1985), 333-348.

[64] P. VÉRTEsI, Hermite-Fejér type interpolations. III, Acta Math. Acad. Sci. Hungar., 34 (1979), 67-84. 
[65] P. VÉRTesi, $\rho$-normal point systems, Acta Math. Acad. Sci. Hungar., 34 (1979), 267-277.

[66] P. VÉRTESI, Lagrange interpolation for continuousfunctions of bounded variation, Acta Math. Acad. Sci. Hungar., 35(1980), 23-31.

[67] P. VÉRTESI, Convergence criteria for Hermite-Fejer interpolation based on Jacobi abscissas, in Foundations, Series and Operators, Proc. of Int. Conf. in Budapest, 1980, v.II, pp.1253-1258, North Holland, 1983.

[68] P. VÉRTESI, One-side convergence conditions for Lagrange interpolation based on the Jacobi roots, Acta Sci. Math. (Szeged), 45(1983), 419-428.

[69] B.D. Vecchia, G. Mastroianni And P. Vértesi, One-side convergence conditions of Lagrange interpolation based on the Jacobi-type weights, Acta Math. Hungar, 99(2003), 329-350.

[70] H. Wang And S. XIAng, On the convergence rates of Legendre approximation, Math. Comp., 81 (2012), 861-877.

[71] H. Wang, D. Huybrechs and S. VAndewalle, Explicit barycentric weights for polynomial interpolation in the roots or extrema of classical orthogonal polynomials, arXiv: 1202.0154, 2013, Math. Comp., to appear.

[72] Y. Wainerman, Some approximating processes connected with the classical orthogonal polynomials, $\mathrm{PhD}$ dissertation, St.Petersburg State University, Russia, 1974 (Russian).

[73] K. WeIERStRASS, Über die analytische Darstellbarkeit sogenannter willkrlicher Functionen einer reellen Veränderlichen, Sitzungsberichte der Akademie zu Berlin 633-639 and 789-805, 1885.

[74] S. XIANG, On the optimal general rates of convergence for quadratures derived from Chebyshev points, arXiv:1308.4322, 2013.

[75] S. Xiang And F. Bornemann, On the convergence rates of Gauss and Clenshaw-Curtis quadrature for functions of limited regularity, SIAM J. Numer. Anal., 50(2012) 2581-2587.

[76] S. Xiang, X. Chen and H. Wang, Error bounds for approximation in Chebyshev points, Numer. Math., 116(2010), 463-491.

[77] S. XIANG AND G. He, The fast implementation of higher order Hermite-Fejér interpolation, SIAM J. Sci. Comput., to appear. 